Purdue University Purdue e-Pubs

$1-31-2008$

\title{
Recent advances in microscale pumping technologies: a review and evaluation
}

Brian D. Iverson

Birck Nanotechnology Center, School of Mechanical Engineering, and Cooling Technologies Research Center, Purdue University, bdiverson@byu.edu

Suresh Garimella

School of Mechanical Engineering, sureshg@purdue.edu

Follow this and additional works at: https:// docs.lib.purdue.edu/nanopub

Iverson, Brian D. and Garimella, Suresh, "Recent advances in microscale pumping technologies: a review and evaluation" (2008). Birck and NCN Publications. Paper 81.

https://docs.lib.purdue.edu/nanopub/81

This document has been made available through Purdue e-Pubs, a service of the Purdue University Libraries. Please contact epubs@purdue.edu for additional information. 


\title{
Recent advances in microscale pumping technologies: a review and evaluation
}

\author{
Brian D. Iverson · Suresh V. Garimella
}

Received: 2 December 2007 / Accepted: 31 January 2008

(C) Springer-Verlag 2008

\begin{abstract}
Micropumping has emerged as a critical research area for many electronics and biological applications. A significant driving force underlying this research has been the integration of pumping mechanisms in micro total analysis systems and other multi-functional analysis techniques. Uses in electronics packaging and micromixing and microdosing systems have also capitalized on novel pumping concepts. The present work builds upon a number of existing reviews of micropumping strategies by focusing on the large body of micropump advances reported in the very recent literature. Critical selection criteria are included for pumps and valves to aid in determining the pumping mechanism that is most appropriate for a given application. Important limitations or incompatibilities are also addressed. Quantitative comparisons are provided in graphical and tabular forms.
\end{abstract}

Keywords Micropump - Microfluidic - Fluid delivery · Electronics cooling $\cdot$ Biofluid

\section{Introduction}

There has been a recent surge in studies exploring micropump technologies, motivated in part by the need to develop pumping mechanisms for biological fluid handling such as for polymerase chain reaction (PCR) and lab-on-achip and micro total analysis systems ( $\mu \mathrm{TAS}$ ) (Zhang et al.

\footnotetext{
B. D. Iverson · S. V. Garimella $(\square)$

NSF Cooling Technologies Research Center,

School of Mechanical Engineering and Birck Nanotechnology

Center, Purdue University, 585 Purdue Mall,

West Lafayette, IN 47907-2088, USA

e-mail: sureshg@ecn.purdue.edu
}

2007). Additionally, micropumps are being considered for application in the cooling of microelectronics as the use of liquid cooling has become increasingly necessary to alleviate the extremely challenging cooling constraints in these compact systems (Garimella et al. 2006; Singhal et al. 2004b). A wide variety of technologies exist for pumping liquids while reducing total pump volume.

Thermal management of electronic components is of increasing concern in the development of portable and reliable electronic devices. The need to reduce package weight and volume while increasing the device functionality has received much attention in recent years. Of the strategies available for thermal management in electronic systems, liquid cooling in microchannels has the ability to increase power dissipation while also maintaining a small form factor. Contact and spreading resistances can be reduced by integrating the channels directly on the back side of common flip-chip designs. Further, by using liquid cooling, the heat generation and heat rejection components can be separated, releasing the convective surface area for ultimate heat rejection to the ambient from being constrained by the microprocessor area (Mahajan et al. 2006). Thus, the heat exchanger in the cooling loop can be placed at any convenient location in the device. However, the requirement of large pumps to drive the liquid flow and the associated large pumping power have limited the application of microchannel heat sinks in space-constrained electronics (Garimella et al. 2006). Innovative micropumping solutions are thus critical for facilitating wider use of liquid cooling approaches in electronic systems.

Strategies for the development of cell and biological analysis tools have also exploited microfluidic devices since they can be used to sample, trap, separate, sort, treat, detect and analyze biological materials (Andersson and Van den Berg 2003). Microfluidic devices offer many 
attractive benefits for biological handling and analysis. For example, reducing device size also reduces sample requirements and reagent volumes, which can reduce overall cost. Test chips are often disposable which is important for sterility. Using microfluidic chips also allows for a closed system, thus protecting the operator from chemical exposure. The small size accommodates parallel operations and thereby reduces cell sorting, analysis and treatment times. Combining different functions on a single microchip is another step toward maintaining a completely closed system that can be fully automated, reduce contamination, and eliminate human intervention and error (Wolff et al. 2003). However, for microfluidic devices to capitalize on all of the above benefits, integration of the fluid pumping mechanism is imperative.

Several extensive reviews of micropump strategies are available (Laser and Santiago 2004; Nguyen et al. 2002; Woias 2005), with some having emphasized specific applications (Singhal et al. 2004b; Zhang et al. 2007). In the present work, past reviews of micropumps are not duplicated. Rather, we provide an overview of pumping mechanisms and important new developments addressed in the recent literature spanning the past few years, and outline the critical selection criteria for determining which of these mechanisms is appropriate for a given application. Micropumping needs vary over a wide range from lowpower, low-flow-rate to high-flow-rate, high-back-pressure solutions. Thus, a variety of pump selection criteria are employed in determining pumping mechanisms suitable for a given application. While the discussion in the text includes quantitative values, a comprehensive quantitative assessment is provided in graphical and tabular forms.

We broadly classify micropumps into two main categories, along the lines of classifications in other reviews:

1. Mechanical displacement micropumps_defined as those that exert oscillatory or rotational pressure forces on the working fluid through a moving solid-fluid (vibrating diaphragm, peristaltic, rotary pumps), or fluid-fluid boundary (ferrofluid, phase change, gas permeation pumps).

2. Electro- and magneto-kinetic micropumps-defined as those that provide a direct energy transfer to pumping power and generate constant/steady flows due to the continuous addition of energy (electroosmotic, electrohydrodynamic, magnetohydrodynamic, electrowetting, etc.).

Micropumps in the above categories can be further divided into sub-categories based on their actuation principle. The following two sections describe the basic working principles of the various kinds of micropumps and valves in the literature. A categorization of the various pump types is shown in Fig. 1. A discussion of these techniques is then provided, wherein we evaluate critical features of various pumps for applicability and sustainability in various applications. Several illustrative figures have been modified from a previous micropump review from our group (Singhal et al. 2004b). For a detailed discussion of studies
Fig. 1 Categorization of pumping mechanisms (topics are discussed in the order presented here)

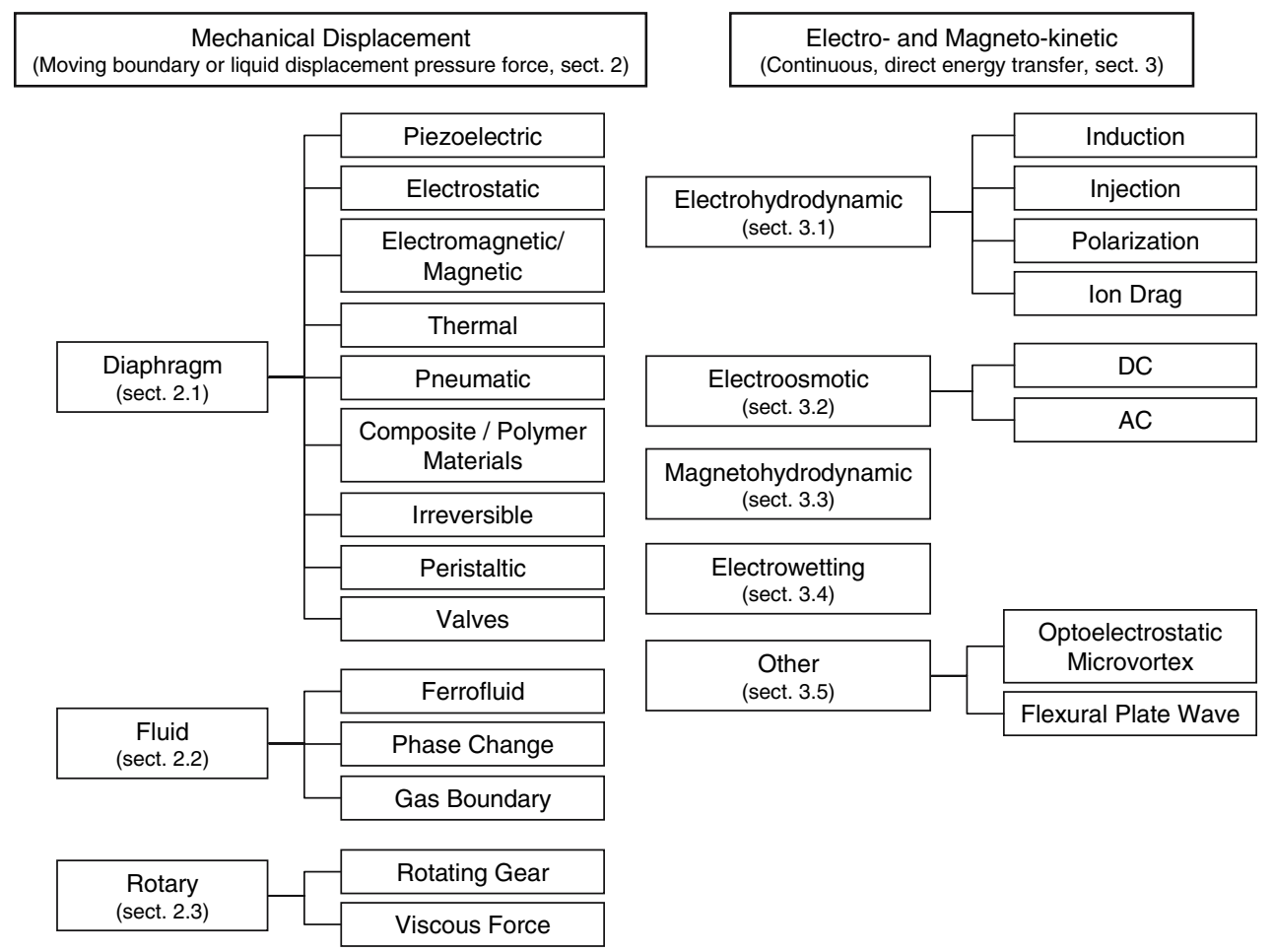


in the literature prior to 2005 , we direct readers to this earlier review.

\section{Mechanical displacement micropumping techniques}

Mechanical displacement micropumps use the motion of a solid (such as a gear or diaphragm) or a fluid to generate the pressure difference needed to move a fluid. Of these, diaphragm pumps are most common and employ many different actuation mechanisms. They also incorporate some type of valving for flow rectification.

\subsection{Diaphragm displacement pumps}

As shown schematically in Fig. 2a, diaphragm displacement pumps typically are comprised of a pumping chamber connected to inlet and outlet valves necessary for flow rectification. As the diaphragm deflects during the expansion stroke, the pumping chamber expands resulting in a corresponding decrease in chamber pressure. When the inlet pressure is higher than the chamber pressure, the inlet valve opens and liquid fills the expanding chamber (Fig. 2b). During the compression stroke, the volume of the chamber decreases with the moving diaphragm, causing the internal pressure to increase whereby liquid is discharged through the outlet valve (Fig. 2c). Many types of actuation mechanisms exist for the vibrating diaphragm. Piezoelectric, electrostatic, electromagnetic, pneumatic, and thermopnuematic actuation are among the more common methods.

(a)

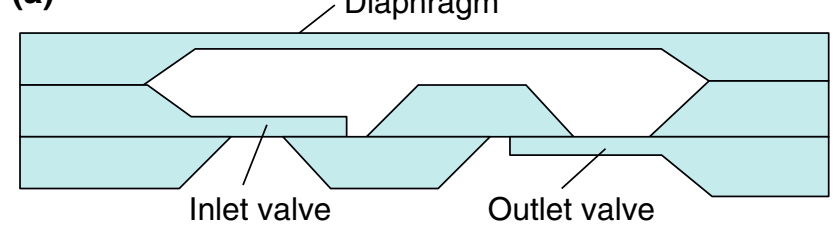

(b)

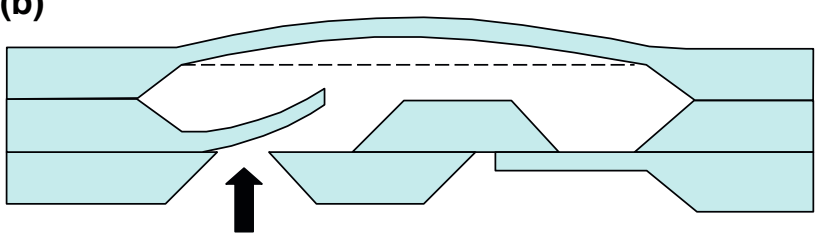

(c)

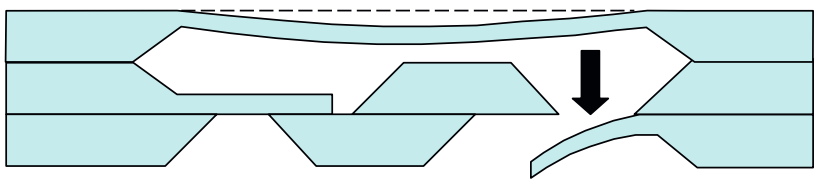

Fig. 2 Vibrating diaphragm micropump in an a undeflected position, and during an $\mathbf{b}$ expansion and $\mathbf{c}$ contraction stroke
Further, valve types differ widely in the literature. Dynamic and static valves have been used for flow rectification and can either be active or passive structures. Diaphragm actuation mechanisms and valve types are explained in the following subsections.

\subsubsection{Piezoelectric}

Piezoelectric materials generate an internal mechanical stress in the presence of an applied electric potential, and vice versa. This mechanism is probably the most widely used for actuating diaphragm micropumps. The piezoelectric material is bonded to, deposited on or embedded in the diaphragm for actuation and the applied AC voltage drives the expansion and compression strokes as the signal changes in polarity. Advantages of this actuation method are that relatively large displacement magnitudes and forces are achievable. Distinguishing characteristics of different piezoelectric micropumps are the chamber geometry, the type of valves used, the shear orientation used for actuation, and the type of piezoelectric material selected.

Standard, flat-diaphragm chamber geometries are fabrication friendly; however, they are less efficient in converting in-plane strain to volumetric deflection as compared to a dome-shaped diaphragm chamber of the same diameter. Further, dome-shaped diaphragms have a higher stiffness, which manifests in higher resonant frequencies. A novel molding process for fabricating domeshaped chambers was developed by Feng and Kim (2005). Their work also illustrates that larger pressures are exerted for smaller-radii pump chambers, which have corresponding larger curvatures.

In order to obtain flow rectification from the oscillatory motion of the diaphragm, valves are incorporated into the design of diaphragm pumps. Valves take many forms and can feature passive or active control. Although a relatively older concept, nozzle-diffuser type fixed-geometry valves have been investigated in the recent past for specific applications such as drug delivery as they employ no moving parts (Cui et al. 2007). Piezoelectrically actuated pumps have become common as they can be fabricated relatively inexpensively, especially when using low-cost, deformable materials such as polydimethylsiloxane (PDMS) (Kim et al. 2005b). Recently, modifications to the standard nozzle-diffuser valves that accommodate regions of vortex circulation (similar to Tesla valves) have been studied for use with piezoelectrically actuated diaphragms (Izzo et al. 2007). These and other valve types are addressed in more detail in Sect. 2.1.9.

Throttling has also been suggested as a potential alternative to conventional valves. Since the volumetric flow rate is inversely proportional to the fourth power of the 
channel hydraulic diameter, small changes in the hydraulic diameter can yield an exploitable modulation of the volumetric flow rate. In order to modulate the hydraulic diameter of the throttle, piezoelectrically actuated diaphragms have been used for semi-passive flow control. The design employed by Tracey et al. (2006) incorporates passive throttle structures in which the hydraulic diameter is actively changing with the piezoelectric diaphragm. Further, their design can accommodate actuation of multiple neighboring channels with the same piezoelectric material. Flow output can be connected in parallel or series depending on whether high volume flow rates or high pressures are desired.

While there is little chance for structural failure of fixedgeometry valves, they do not always provide the necessary flow rectification for higher flow rates and cannot operate in a bi-directional mode. Active diaphragm valves that can overcome such shortcomings are also commonly controlled with piezoelectric diaphragms (Doll et al. 2006). In these scenarios, the piezoelectrically actuated diaphragms are used not only for the pump chamber but also to open and close the inlet/outlet valves in sync with the chamber expansion and compression.

A lightweight piezoelectric composite actuator (LIPCA) has been reported as an improvement to traditional piezoelectric actuators (Nguyen et al. 2006). This oxide-based, piezoelectric material incorporates carbon or glass and epoxy fabric. Experimental results with a PDMS diaphragm show that the fractional stroke (displacement per unit length) of the LIPCA is approximately $0.35 \%$ as compared to conventional piezoelectric diaphragms which are normally associated with values lower than $0.2 \%$. Thus, comparatively larger displacements can be achieved with this material for the same driving potential.

Novel piezoelectric diaphragm actuation using shear deformation has also recently been considered in microfluidic applications (Chen et al. 2007). Actuation in this orientation can be used for droplet ejection and diaphragm pumping applications.

A complete electro-mechanical-fluid coupled model of a piezoelectric-actuated, diaphragm pump demonstrated that flow rate should not increase continuously with increasing frequency for high frequencies (Fan et al. 2005). Optimization studies have also been conducted for both membrane valves and nozzle-diffuser valves using equivalent electrical networks for representation and modeling in SPICE (Morganti et al. 2005).

\subsubsection{Electrostatic}

Electrostatic actuation employs the use of electrostatic forces generated between electrodes to drive diaphragm motion (Zengerle et al. 1992). As an electrical voltage is applied between a counter electrode and the diaphragm membrane, they act as a variable capacitor and the electrostatic forces generated cause movement of the membrane outward, towards the counter electrode. Hence, the pressure in the pumping chamber decreases, drawing fluid into the chamber. When the voltage is removed, the membrane bounces back, which increases the pressure in the pumping chamber, thus expelling the fluid. The capacitance between a pump diaphragm and a counter electrode (diameter, $d$ ) separated by a distance $(l)$ can be calculated by

$C=\frac{\varepsilon \pi d^{2}}{4 l}$.

The force acting to pull the two plates together is

$F=\frac{1}{2} \frac{\partial C}{\partial l} V^{2}=-\frac{\varepsilon \pi d^{2}}{8 l^{2}} V^{2}$

where $V$ is the potential difference across the plates, and $\varepsilon$ is the permittivity.

Electrostatic actuation has received comparatively little attention in the recent micropump literature. However, an advancement over the typical electrostatic actuation described above has been considered in which voltage is applied across the liquid working fluid to take advantage of the higher relative electrical permeability of water/fluids as compared to air (Machauf et al. 2005). The higher the permittivity, the higher are the force and the pumping rate for the same applied voltage and geometry. Thus, even for relatively large distances between electrodes, the generated force across the liquid can be large enough to induce pumping. Although not an ideal design, the experiments of Machauf et al. (2005) showed that approximately $1 \mu \mathrm{l} / \mathrm{min}$ could be pumped at $50 \mathrm{~V}$ (across $63 \mu \mathrm{m}$ gap) under suboptimal conditions.

Pumping using electrostatic actuation was developed for a gas chromatograph application (Astle et al. 2007). Improved understanding of the gas flow in the micropump was one of the major aims of this work. A four-stage pump operating at $14 \mathrm{kHz}$ provided a flow rate of $3 \mathrm{ml} / \mathrm{min}$ and a maximum back pressure of $7 \mathrm{kPa}$.

\subsubsection{Electromagnetic and magnetic}

The electromagnetic actuation mechanism generally consists of a permanent magnet attached to a diaphragm and surrounded by a coil. When a current is passed through the coil, Lorentz forces are produced to deflect the diaphragm due to the interaction of the magnetic field with the electric field. Advantages of this method are that electromagnetic actuation generally requires a small voltage $(\sim 5 \mathrm{~V})$ and 
has a simple design of driver electronics as compared to other mechanisms. This type of micropump has been discussed in the literature for years. Recently, diaphragm deflections have improved for micropumps with overall size reductions achieved by integrating the permanent magnet and coils directly into the device (Chang et al. 2007; Su et al. 2005; Yufeng et al. 2006).

A permanent magnet cast in PDMS with flow rectification provided by ball valves was developed by Yamahata et al. (2005b). The conical holes for the ball valves were powder blasted in glass and the device was fusion-bonded together. The glass construction (valves and body) is an important feature as it is chemically inert and can be sterilized at high temperatures. Typically, glass devices do not provide high back pressures which are achieved by large actuation forces in combination with highly efficient valves. However, a maximum flow rate of $5 \mathrm{ml} / \mathrm{min}$ was demonstrated at $30 \mathrm{~Hz}$ with a maximum back pressure of $28 \mathrm{kPa}$ for a diaphragm chamber diameter of $1 \mathrm{~cm}$.

A similar ball-valve-based, magnetically driven micropump was investigated using a magnet attached to a DC motor, and alternatively using an inductive coil (Pan et al. 2005). A large reduction in power requirement was demonstrated in the DC motor-driven case $(13 \mathrm{~mW})$ as compared to the coil $(500 \mathrm{~mW})$ for comparable flow rates. The ball valves help to limit backward leakage to less than $1 \mu \mathrm{l} / \mathrm{min}$ for pressures of up to $30 \mathrm{kPa}$ and maximum flow rates of $0.7-1 \mathrm{ml} / \mathrm{min}$.

Construction of composite diaphragms in which small magnetic particles are cast directly into the PDMS have also been developed. In this manner, the diaphragm boundary directly incorporates the material for actuation by the magnetic field. Nagel et al. (2006) used an iron-PDMS (Fe-PDMS) composite with less than $10 \mu \mathrm{m}$ diameter iron particles. Yamahata et al. (2005c) used a $40 \%$ volume fraction of $\mathrm{NdFeB}$ powder (average particle size $200 \mu \mathrm{m}$ ) in PDMS for a membrane stroke of $200 \mu \mathrm{m}$. Nozzle-diffuser elements were incorporated for flow rectification.

Other types of magnetically induced flows have been achieved by exerting attractive forces between a permanent magnet and steel disks incorporated into pumping and valve chambers (Haeberle et al. 2007). In this design, the permanent magnet is mounted on a rotating disk. As the disk rotates and the magnet passes by the static steel disks in the valve and the pumping chamber diaphragms, the chambers contract and expand in succession. The appeal of this design is that the only power required to drive this pump is the relatively low power required for the disk rotation.

Magnetic fluids have emerged as an additional choice for micropump actuation. Fluids of this type are generally a suspension of magnetic particles in a carrier medium. Sim et al. (2006) used the response of a magnetic fluid to a field to actuate a parylene diaphragm in a micropump. The salient features of this pump are that at a field of 110 Gauss, the diaphragm could be deflected by more than $200 \mu \mathrm{m}$ to provide a pressure of $2.8 \mathrm{kPa}$. The suspended magnetic particles, which have an average size of about $100 \AA$, are coated with a stabilizing dispersant to prevent agglomeration in the presence of a magnetic field. The suspensions are stable and preserve their properties despite exposure to extreme temperatures and over long periods of time.

\subsubsection{Thermal}

Thermal actuation involves the volume expansion or induced stress of a material in response to applied heat. In the context of diaphragm micropumps, this usually takes the form of thermopneumatic or shape memory alloy (SMA) actuation. Since these methods rely on the diffusion of thermal energy, they are limited to low actuation frequencies.

Thermopneumatic actuation occurs when a secondary fluid (separate from the driven fluid) is heated (usually by a thin film resistive heater) causing it to expand and deflect the pump diaphragm. The intake stroke (pump chamber expansion) occurs as the heater is deactivated allowing the secondary fluid to cool and contract. A transparent, costeffective thermopneumatic pump using PDMS and nozzlediffuser elements was developed by Kim et al. (2005a). Usage of indium tin oxide (ITO) as the conductive heating element provides transparency if desired. For a diaphragm diameter of $3.5 \mathrm{~mm}$ and inlet/outlet lengths of $1.5 \mathrm{~mm}$, a peak flow rate of $78 \mathrm{nl} / \mathrm{min}$ was demonstrated for methanol with an applied pulse of $55 \mathrm{~V}$ at $6 \mathrm{~Hz}$.

Thermal expansion also occurs for phase-change materials as opposed to single-phase gas expansion. Paraffin waxes, also used for transient heat absorption from electronics, have been employed to actuate a diaphragm pump by exploiting its volume expansion from solid to liquid as it melts due to resistive heating. Melting temperatures for paraffin can be tailored to lie between -100 and $150^{\circ} \mathrm{C}$. The material can also be shaped by casting, and is nontoxic. Since it is a thermal actuator, the actuation frequency is relatively low; however, it can sustain very high pressures. Boden et al. (2006) used Sigma-Aldrich 76228 with a melting point of $44-48^{\circ} \mathrm{C}$ and a volume expansion of about $10 \%$ to obtain flow rates of $74 \mathrm{nl} / \mathrm{min}$ and up to $0.2 \mathrm{MPa}(0.92 \mathrm{MPa}$ with clamping) at $0.5-\mathrm{Hz}$ frequency.

Shape memory actuation uses the shape memory effect of TiNi, which involves a phase transformation between two solid phases: a high-temperature austenite phase and a low-temperature martensite phase. Martensite is much more ductile than austenite allowing the TiNi to undergo large deformations. Heating above the phase-transformation 
temperature results in an austenite phase transition in the TiNi. During this transformation, TiNi assumes its initial shape if it is not constrained. However, when constrained, it exerts a large force in trying to assume its initial shape. Shape Memory Alloys (SMAs) are characterized by large recoverable strain outputs of up to $6-8 \%$. However, at high frequencies, SMAs do not cool sufficiently and their performance suffers. Generally, they operate below $100 \mathrm{~Hz}$.

Recently, larger flow rates of $2.53 \mathrm{ml} / \mathrm{s}$ have been achieved for a thin-film TiNi SMA pump with corresponding velocities of $5 \mathrm{~mm} / \mathrm{s}$ (Shin et al. 2005). This flow rate represents a three-order-of-magnitude increase over previously published SMA pump studies. Another SMA pump with a $\operatorname{TiNi}(\mathrm{Cu})$ alloy has demonstrated low temperature actuation (Zhang and Qiu 2006). In some applications, the working fluid in the pump chamber is to be kept below a specific temperature in order to not adversely affect the fluid. Using this $\mathrm{TiNi}(\mathrm{Cu}) \mathrm{SMA}$, actuation below $290 \mathrm{~K}$ is achieved while providing a high diaphragm displacement $(6 \mu \mathrm{m})$ and relatively high working frequency $(85 \mathrm{~Hz})$.

\subsubsection{Pneumatic}

Pneumatic pumps exploit fluctuations in gas pressure on a diaphragm to effect vibration. As gas pressure builds on the diaphragm, deflection occurs for the compression stroke. Pneumatic valves are actuated in a similar manner for flow rectification. Pneumatic driving forces are commonly employed in a peristaltic actuation sequence as is discussed in more detail in Sect. 2.1.8.

Diaphragm pumps are generally characterized by a pulsing flow due to the reciprocating nature of the boundary. A bi-directional, pneumatic diaphragm pump that incorporates a fluidic capacitor to convert the pulsatile flow into a continuous stream was developed by Inman et al. (2007). For a pneumatic pressure of $40 \mathrm{kPa}$, a $2.6 \mathrm{ml} /$ min flow rate was achieved against a $25 \mathrm{kPa}$ back pressure.

\subsubsection{Composite/polymer materials}

Materials selection, to a great extent, distinguishes diaphragm micropumps. Composite materials have been developed as improved alternatives to some of the commonly used actuation materials. An ionic polymer-metal composite (IPMC) material was electromechanically actuated, similar to piezoelectric materials, to create a larger bending deformation (over $1 \%$ bending strain) under a low input voltage by (Lee and Kim 2006; Lee et al. 2005). The manufacturing costs of this composite are stated to be competitive with other actuator technologies. As compared to piezoelectrically driven diaphragms, IPMCs require significantly lower input voltages thus making them attractive as a driving mechanism.

Another diaphragm material has been demonstrated to compete well with traditional diaphragm actuating mechanisms. An electroactive polymer (polyvinylidene fluoridetrifluoroethylene-PVDF TrFE) has achieved displacements of $21 \mu \mathrm{m}$ on a $1 \mathrm{~mm}$ diameter diaphragm at $106 \mathrm{~V} /$ $\mu \mathrm{m}$ and a driving frequency of $10 \mathrm{~Hz}$ (Xu and Su 2005). The unstretched PVDF films make film processing very simple and the isotropic strain response is amenable to circular shaped actuators. Further, these materials are characterized by higher strains than are common in piezoelectric ceramics.

\subsubsection{Irreversible}

Actuation mechanisms in which the induced deflection is irreversible (and hence, not cyclic) are denoted as irreversible approaches. However, irreversible pumps have many attractive features despite this limitation. Generally they require no input power for actuation and, in some cases, can produce large pressures. When maximum portability is required and the use of electrical networks or power is disallowed, these technologies are particularly suitable.

Ionic polymer particles that swell due to osmotic effects have been demonstrated to be viable for liquid pumping. The swelling is induced simply by adding water (Good et al. 2007). Gels also can be engineered to deform under some physical or chemical stimulus and used as an actuator for irreversible diaphragm deflection (Suzuki 2006).

Thermally responsive pumping has also been achieved through the use of expandable microspheres. Composites of these microspheres in PDMS have been constructed such that as the material is heated, the embedded microspheres expand the material up to $270 \%$. Again, the expansion is irreversible but has been shown to propel nanoliters of flow against $100 \mathrm{kPa}$ back pressures (Samel et al. 2007a, b). Larger volumes on the order of microliters have also been moved by buckling the PDMS composite. In instances where the trigger mechanism is a rise in temperature, these materials are directly applicable since there would be no transduction or consumption of external energy for actuation.

The irreversible actuation principles discussed here are likely to be used only for single-use, disposable applications.

\subsubsection{Peristaltic}

As the name suggests, these pumps incorporate the peristaltic motion of actuators in series to generate pumping 
action. Most of the peristaltic pumps presented in the literature use three pumping chambers with diaphragms as actuators in series (see Fig. 3). Thus they can be considered a subset of the vibrating diaphragm pumps considered above, and they utilize many of the same types of transducers (piezoelectric, pneumatic, etc.). When the first diaphragm is actuated, it restricts the flow to the inlet of the pump. As the second diaphragm is actuated, fluid is pushed toward the third pumping chamber. Similarly, actuating the third diaphragm in succession pushes the fluid through the outlet of the pump. In essence, the diaphragms act like valves that reduce the flow cross section to provide flow directionality. All three diaphragms are then de-actuated and the sequence is repeated continually for pumping action from left to right. However, reverse order actuation makes peristaltic pumps bi-directional.

Peristaltic pumps continue to be investigated in the recent literature. One of their advantages is that they can provide comparatively high-back pressures (Geipel et al. 2007; Jang et al. 2007; Lin et al. 2007). Flow rate measurements on a range of pneumatically actuated, peristaltic, PDMS pump geometries have been presented along with a simple non-linear model to describe the pump dynamics (Goulpeau et al. 2005). For a pneumatic pressure of $20 \mathrm{kPa}$ they were able to achieve a flow rate of $7.5 \mu \mathrm{l} / \mathrm{min}$ at $250 \mathrm{~Hz}$. A similar three-stage, thermopneumatic, PDMS, peristaltic pump achieved $21.6 \mu \mathrm{l} / \mathrm{min}$ using $20 \mathrm{~V}$ to heat the air in a sealed actuation chamber (Jeong et al. 2005).

An analytical model for micro-diaphragm pumps with active valves based on the peristaltic working principle has been developed (Goldschmidtboing et al. 2005). It applies
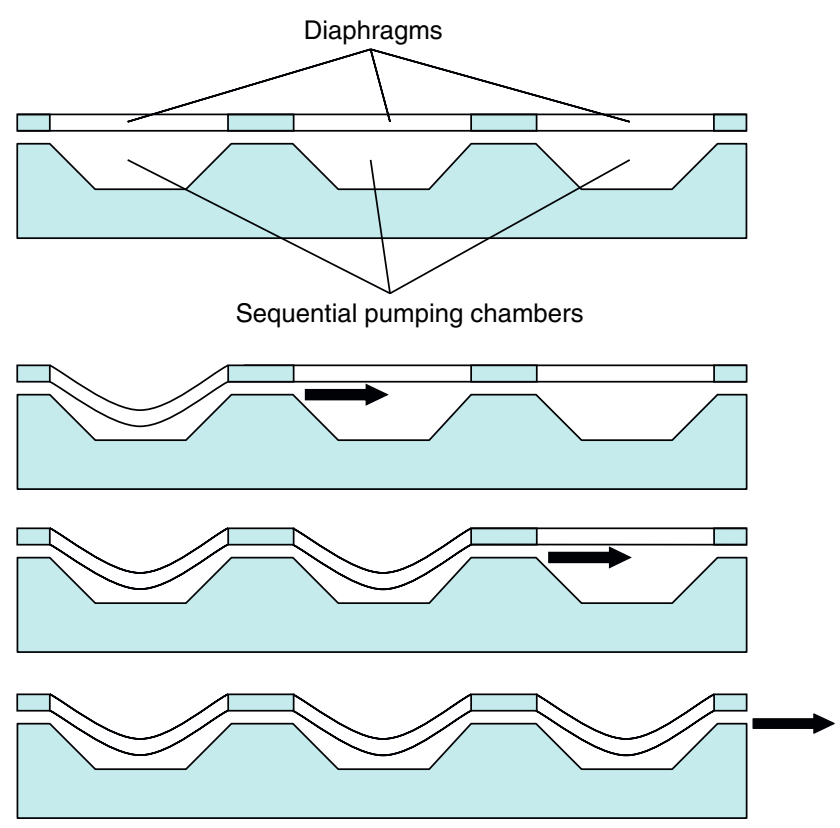

Fig. 3 Structure and operation of a peristaltic micropump to both fast and slow actuation mechanisms such that it can be used for piezoelectric, pneumatic, thermopneumatic and other driving mechanisms. They show that micro-diaphragm pumps suffer from a linear dependence on the flow rate with applied back pressure.

Magnetic fluids have also been considered as an actuation mechanism for peristaltic pumping. Magnetic fluid is attracted and gathered using a permanent magnet (controlled by a stepping motor) into a round-shaped accumulation, which deforms the silicone rubber diaphragm (Kim et al. 2006). These lumps are then manipulated by the magnetic field to pump liquid in a peristaltic fashion yielding a maximum flow rate of $3.8 \mu \mathrm{l} /$ min.

Single-source-actuated peristaltic pumps have been proposed in recent years. The general design consists of several pumping chambers that are connected serially such that the time-phased deflection of the successive membranes generates a peristaltic effect. With the drive chambers connected, they can be controlled by a single source, thereby reducing the potential for failure of components. However, these peristaltic pumps are unidirectional. A single electromagnetic valve and pneumatic source was incorporated into a three-chamber design by Huang et al. (2006a). A similar pump actuated by a single pneumatic source employed a serpentine pneumatic channel where the intersection of the pneumatic channel and fluid channel constituted areas of membrane deflection (Yang et al. 2006). Seven intersections provided seven stages of peristaltic actuation. They showed that the flow rate could be increased by increasing the pneumatic pressure, operational frequency or number of membranes (intersections). These s-shaped pneumatic, peristaltic pumps have been used effectively in cell sorting and cytometry applications.

\subsubsection{Valves}

Reciprocating diaphragms require some sort of flow rectification in order to produce net flow. Diaphragm displacement profiles are generally symmetric resulting in non-directional flow. Hence, valves are used to convert the non-directional flow to directional flow. Valves can be classified into dynamic- and static-geometry categories, and further divided into active or passive sub-categories. We distinguish the term "valveless" pumps from static or fixed-geometry valves; specifically, valveless pumps do not have components that provide flow directionality (usually because the pumping mechanism has inherent directionality), while static and fixed-geometry valves do have such components even though they may not incorporate moving parts. 
2.1.9.1 Dynamic geometry Dynamic-geometry valves are defined as structures that provide flow direction by deformation, motion or deflection. Active valves are those that require energy (electrical, thermal, etc.) for flow rectification. Almost any of the diaphragm actuators discussed for vibrating diaphragm pumps (Sect. 2.1) can be used as an active, dynamic valve. These valves operate by opening and closing in sync with the diaphragm vibration such that the pump chamber outlet is closed before the expansion stroke and the pump chamber inlet is closed before the compression stroke (Fig. 4). Hence, the flow becomes directional. Cantilever structures (similar to those in Fig. 2) are also commonly used as valve structures and have been shown to be amenable to active control using piezoelectric and RF modulation (Dissanayake et al. 2007).

Thermally responsive valves can also be made with PDMS by casting thermally expanding microspheres in the PDMS (Samel et al. 2007b). As the temperature increases, the microspheres swell and designs can be conceived whereby the swelling action is used to close a valve. While this type of valve does require thermal energy for activation, it can also be considered passive if the thermal energy required for closure is inherent to the system operation. Despite the irreversible nature of this thermally actuated valve, the expansion has been shown to hold pressures of up to $140 \mathrm{kPa}$ without any required electrical input.

Dynamic-geometry valves can also be passive in that they require no energy for activation. Again, flexible cantilever structures have been used (similar to Fig. 2). Since PDMS is highly flexible, it has been used extensively as a dynamic, passive valve. Valve designs for these flap structures differ when considering low and high Reynolds number flows. Further, consideration must be given to adhesion between the PDMS flap and its seat so as to prevent stiction (Loverich et al. 2007).

The passive nature of ball valves may suggest their use with micropumps. Ball valves are excellent candidates for the generation of unidirectional pumping flows, though they have been rarely used in micropumps. This is likely
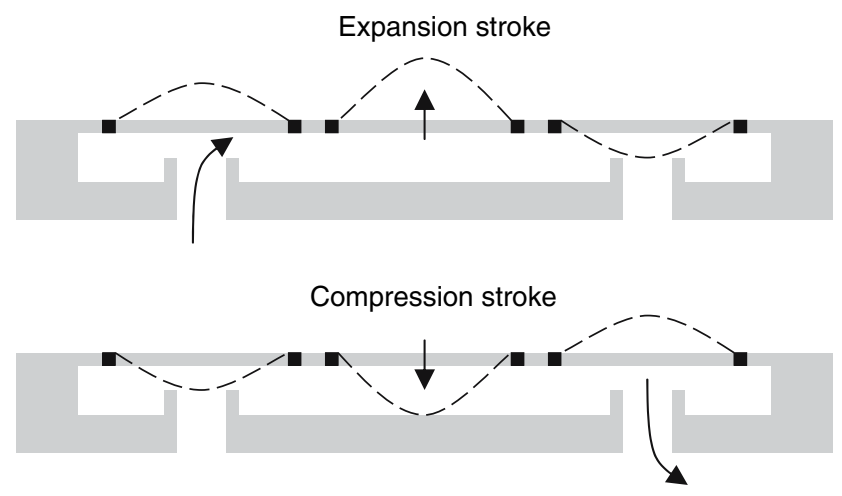

Fig. 4 Dynamic, active diaphragm valves due to the difficulty in combining them with classical twodimensional microfabrication techniques. Recently, however, conical holes for the ball valve seat have been fabricated using a powder blasting technique (Yamahata et al. 2005b). Maximum-back pressures up to $28 \mathrm{kPa}$ have been demonstrated with a device employing these valves. Ball valve seats made from micropipettes have also been constructed with similar back pressures of up to $30 \mathrm{kPa}$ (Pan et al. 2005).

Dynamic-geometry valves run the risk of fatigue failure in long-life operation. Stiction can also prove to be problematic when the valve does not release from its seat. Further, their dynamic nature has an inherent response time required for activation in response to a change in flow direction. This time requirement must be included in determining operating frequencies. However, since dynamic-geometry valves commonly provide a physical barrier to reverse flow, they often can withstand large-back pressures. Mechanical displacement micropumps commonly incorporate some version of a normally closed, passive, mechanical flap structure as a valve. Flap valves based on cantilever structures are easily fabricated and widely used.

2.1.9.2 Static geometry By definition, static-geometry valves employ no moving parts or boundaries for flow rectification. Rather, the geometry is fixed and the conversion of non-directional flow into directional flow occurs through the addition of energy (active) or through geometries in which the desired flow behavior is induced by fluid inertia (passive). Static-geometry valves have been extensively used due to the simplicity of their design and the low risk of failure.

Among the static-geometry valves that are actively controlled, laser-induced heating that generates thermocapillary stress at the interface of two immiscible fluids has been employed to restrict the inflow of liquid in microchannel crossflow (Baroud et al. 2005). Localized heating reduces the surface tension at the point of heating. The surface-tension imbalance induces flow along the interface from the point of low surface tension (high temperature) to the region of high surface tension (low temperature), also known as Marangoni flow. This effect is amplified in miniaturized systems since temperature and surface tension gradients are increased with smaller length scales. While this localized heating has been shown to provide active valving, it can also move droplets of water in oil with no moving parts.

Thermally controlled, viscosity-based valves have also been investigated in vibrating diaphragm pumps (Matsumoto et al. 1999). The rectification principle is based on the temperature dependence of liquid viscosity, which 
causes a variation in flow resistance. The structure of a micropump with viscosity-based valves is illustrated in Fig. 5a. The pump chamber with a vibrating diaphragm is connected to inlet and outlet channels through small, crosssectional chokes with Boron-doped silicon heaters for local heating. As the diaphragm compresses the pump chamber volume, the outlet choke is simultaneously heated such that the viscosity of the liquid decreases near the outlet and, hence, more liquid exits through the outlet during the compression, and vice versa.

Static-geometry, passive valves rely on the geometry itself to produce directional flow. The two most common types are nozzle-diffuser and Tesla valves. However, structures for throttling flow have also been used as a valve mechanism. This genre of valves is very attractive since there are no moving parts and they require no additional energy for operation. Hence, they are the least likely to fail.

Parallel- and perpendicular-geometry nozzle-diffuser valves are illustrated in Fig. 5b and c, respectively. During the expansion stroke of actuation, the inlet region acts as a diffuser and the outlet acts as a nozzle for the liquid flowing into the pump chamber. Hence, more fluid enters the chamber from the inlet side than the outlet side. Conversely, during the compression stroke the inlet region acts as a nozzle and the outlet acts as a diffuser resulting in more fluid being expelled to the outlet side. In this manner, net flow is generated from the inlet to the outlet. The concept was first presented by Stemme and Stemme (1993). Fixed-geometry nozzle-diffuser valves for use with low Reynolds number flows were studied by Singhal et al. (2004a) demonstrating their ability to rectify flow for laminar flows with the larger rectification occurring at higher Reynolds numbers.

Tesla valves (shown in Fig. 5d) are bifurcated channels in which the separated flow re-enters the main flow channel perpendicularly when the flow is in the reverse direction. The idea was first conceptualized in 1920 (Tesla 1920) and has been used in many micropumps over the years. An optimization study has been conducted using six independent non-dimensional geometric design variables in a numerical study to optimize the Tesla valve shape (Gamboa et al. 2005). Rectification improvements of $25 \%$ were achieved by simple geometry modifications without any increase to forward flow resistance. Tesla valves have been used on a piezoelectric actuated, diaphragm pump for use in a thermal management system (Faulkner et al. 2006).

The combination of nozzle-diffuser and Tesla type valves was investigated by Izzo et al. (2007). Specifically, regions for vortex circulation (similar to Tesla valves) were added along the sides of the nozzle-diffuser regions for flow rectification.

The use of throttles has also been used to replace conventional valves in micropump structures. Since the volumetric flow rate is inversely proportional to the fourth power of the channel hydraulic diameter, even small hydraulic diameter changes can effectively modify the volumetric flow rate (Tracey et al. 2006). Although the pump chamber diaphragm vibrates during operation, thus changing the cross-sectional area in the valve region, we designate these valves as static and passive simply because the diaphragm actuation used for pumping is being exploited to provide the hydraulic diameter changes as opposed to changes in the valve structure.

There are a number of considerations in selecting a valve type for a specific application. First, static- or dynamic-geometry valve designs are selected based on the desired level of flow rectification. Second, one must consider whether the potential advantage in flow rectification for actively powered valves is worth the added complexity and power consumption over that for passive valves. The
Fig. 5 Static, fixed-geometry valves: a thermally controlled viscosity valve, b, c nozzlediffuser valves and $\mathbf{d}$ Tesla valve (a)

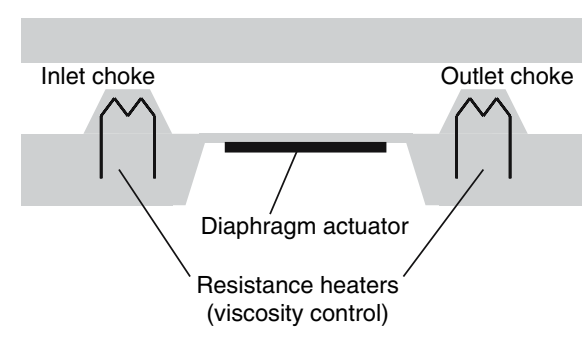

(c)

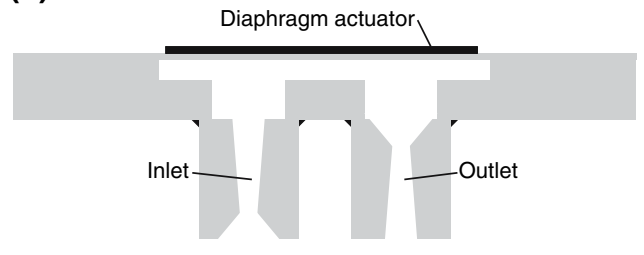

(b)

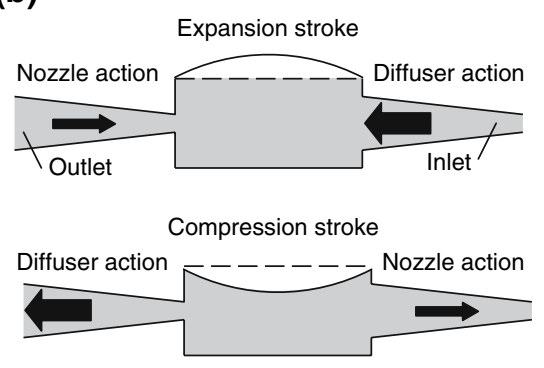

(d)

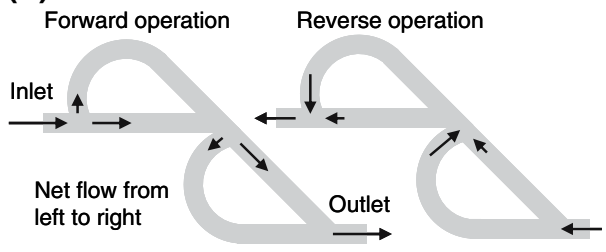


absence of moving parts in fixed-geometry valves can be especially advantageous when the fluid contains cells or other materials that may clog. Further, they eliminate wear and fatigue issues inherent in cantilever or dynamicgeometry valves. Static-geometry valves are generally not bi-directional which can be problematic for flexibility in biological detection and analysis systems. In some cases, it is advantageous to repeatedly move fluid back and forth across a region to increase biological binding events and take advantage of the agents present in the fluid. In particular, nozzle-diffuser and Tesla valves provide directiondependent flow resistance.

\subsection{Fluid displacement pumps}

Fluid displacement pumps are characterized by the direct manipulation of the working fluid by a secondary fluid without the use of a diaphragm. The driving fluids are in direct contact with the working fluid and therefore must be immiscible. In the case of liquid displacing liquid, ferrofluids are commonly used as the actuating mechanism. In the case of gas displacing liquid, actuation mechanisms include phase change and gas boundary work.

\subsubsection{Ferrofluid}

Ferrofluids have been used to directly displace fluid without a diaphragm. A ferrofluidic plug in a y-shaped channel with two passive check valves was demonstrated as a micropumping option (Yamahata et al. 2005a). The ferrofluid is water-based and separated from the working fluid with an oil plug. Actuation of the ferrofluid is performed by the linear periodic motion of an external permanent magnet, thereby giving rise to a ferrofluidic piston. Maximum flow rates of $30 \mu \mathrm{l} / \mathrm{min}$ and back pressures of $2.5 \mathrm{kPa}$ were reported.

\subsubsection{Phase change}

Phase change micropumps utilize volume changes from phase transition to displace fluid for pumping. Usually, this takes the form of liquid-to-vapor phase change because of the significant increase in volume. Bubble pumps and electrochemical pumps are common to this category of pumps.

Ordinary bubble pumps usually consist of independently controlled heaters along a closed microchannel as shown in Fig. 6. Initial heating at the first stage occurs for a sufficiently long time so as to initiate and grow a vapor bubble to fill the channel cross-section. Activation of a second
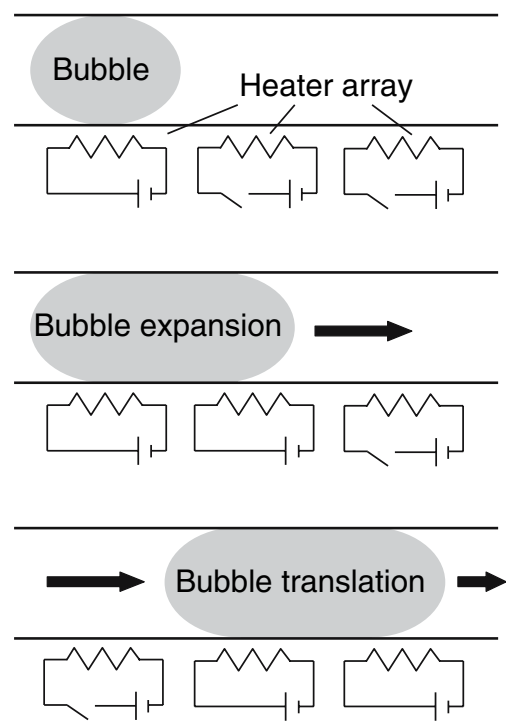

Fig. 6 Bubble micropump with sequential bubble growth stages

heater causes a gradient in the vapor pressure of the bubble. This, along with the gradient in surface tension results in a streamwise pressure gradient. Hence the bubble moves from left to right and the motion is sustained by deactivating the first stage and activating the third stage, and so on. The fluid is displaced with traveling vapor as the bubble covers the channel cross-section. By comparison, bubble pumps can be high-energy consumers since the latent heat of vaporization is commonly provided by resistive heating; however, their device architecture is extremely simple and can be fabricated in a small footprint. Bubble pumps have been developed for conducting fluids (Yin and Prosperetti 2005b) and non-conducting fluids (Yin and Prosperetti 2005a) with flow rates on the order of $1-200 \mu \mathrm{l} / \mathrm{min}$.

Unlike the sequential heating of sections in the lengthwise direction, nozzle-diffuser inlets and outlets have also been proposed in order to provide flow rectification to an expanding bubble in a pumping chamber (Jung and Kwak 2007). These pumps provided flow rates comparable to other bubble pumps, on the order of $1-10 \mu \mathrm{l} / \mathrm{min}$.

The basic design of an electrochemical pump consists of a pair of closely spaced electrodes in a small reservoir filled with water. The reservoir is connected to a channel filled with the liquid to be pumped. When a voltage difference is applied across the electrodes, the water breaks down into its components of oxygen and hydrogen forming gas bubbles by electrolysis. The bubbles are then used to push the liquid in the channel to induce flow (see Fig. 7). A recent paper exploiting this mechanism used electrochemical reaction not only to drive fluid flow but also to deflect a diaphragm used for a valve (Lee et al. 2007). 


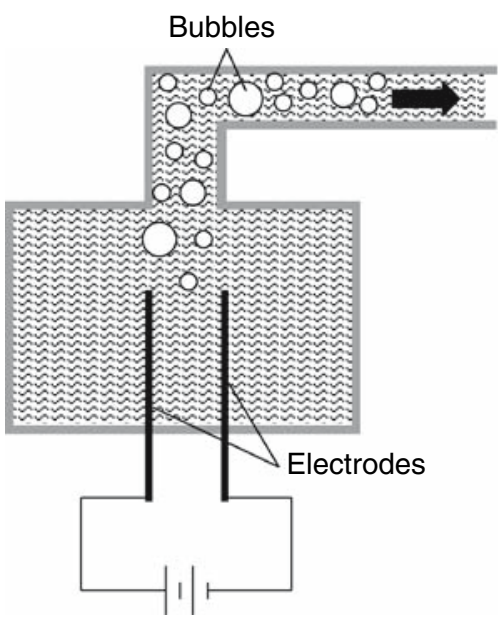

Fig. 7 Electrochemical micropump schematic

\subsubsection{Gas boundary work}

Gas contraction and permeation pumps are characterized by a gas boundary performing work directly on the liquid to be driven. Thermopneumatic pumps without a diaphragm are included in this category, since they capitalize on the work performed by volumetric expansion or contraction of gases. Also, gas permeation through a boundary can perform work as gas accumulation or removal displaces the liquid.

A single-stroke thermopneumatic pump actuated without diaphragm displacement was demonstrated to produce a $0.34 \mu \mathrm{l} / \mathrm{min}$ flow rate (Song and Lichtenberg 2005). Thermal expansion of air in a pump chamber $(16 \mu \mathrm{l})$ with a single inlet is used to manipulate the fluid. Liquid is drawn into the channel in a single stroke. It is pulsation-free, incorporates no moving parts, and has a very low fabrication cost.

A bi-directional pump operating by gas diffusion across a permeable PDMS membrane was developed by Eddings and Gale (2006). Either an applied pressure or vacuum can be used to move the fluid. The pump was capable of directing flow through networks, and could produce bubble-free, fluid-filled dead-end channels or chambers. When a vacuum is applied, air is pulled through the PDMS membrane allowing the filling of dead-end channels or the removal of air bubbles. This method is also a viable replacement to the channel outgas technique in which a microdomain is filled with fluid by submerging the device in the working fluid and then placing it in a vacuum environment.

\subsection{Rotary pumps}

Traditional rotary micropumps consist of a toothed gear rotating in a fluid chamber with an inlet and an outlet port.
However, there have been several additional micropumps in which the fluid is driven with a rotating component, either internal or external to the fluid flow path. The following sections describe these "rotating-gear" and "viscous-force" pumping mechanisms.

\subsubsection{Rotating gear}

Typically rotating-gear micropumps drive the finned or toothed gear with an electric motor for rotation. Fluid becomes entrapped between the gear teeth while turning and thereby is transported from the inlet to the outlet. Figure 8a illustrates such a device. Other versions of this design incorporate multiple, enmeshed rotating gears. A traditional rotating shaft pump with fins was reported to have achieved a flow rate of $9.5 \mathrm{ml} / \mathrm{min}$ for only $0.165 \mathrm{~W}$ power input (Lei et al. 2007).

Dual rotating lobes, cooperatively driven by means of the time-divided scanning of a single laser beam, have been shown to produce very low flow rates on the order of $1 \mathrm{pl} /$ min (Maruo and Inoue 2006). The lobes are driven by means of radiation-pressure generated by focusing a laser beam. The rotor can be controlled by changing the trajectory of the scanning laser beam. A toothed, dual-gear micropump fabricated using LIGA technology was shown to provide relatively large flow rates (up to $8.5 \mathrm{ml} / \mathrm{min}$, $3 \mathrm{~mm}$ diameter gears) with capability of high-back pressures (up to $9.8 \mathrm{kPa}, 2 \mathrm{~mm}$ diameter gears) (Matteucci et al. 2006).

\subsubsection{Viscous force}

Fluid displacement using viscous forces generated by a rotating component has been investigated by several researchers, each with different configurations (illustrated in Fig. 8b-d). These concepts have been developed relatively recently and are yet to be employed in specific, multi-functional applications.

Eccentric placement of a rotating shaft in a straight channel has been presented as a potential micropump mechanism. When the cylinder rotates, a net force is transferred to the fluid due to unequal shear stress on opposite sides of the rotor (see Fig. 8b). Numerical investigations of this pump type have been performed along with the fabrication of a larger scale version $(\mathrm{cm}$ scale) for validation of their numerical code (Yokota et al. 2006). A similar study developed a numerical model of multiple rotors in various configurations for straight channel geometry (Abdelgawad et al. 2005). They showed that dual-vertical rotor configurations yield the best efficiencies and the highest flow rates. Asymmetric placement 
Fig. 8 Principle of operation of a rotating blade, and $\mathbf{b}$ eccentric viscous, c spiral-channel viscous, and $\mathbf{d}$ disk viscous micropumps (a)

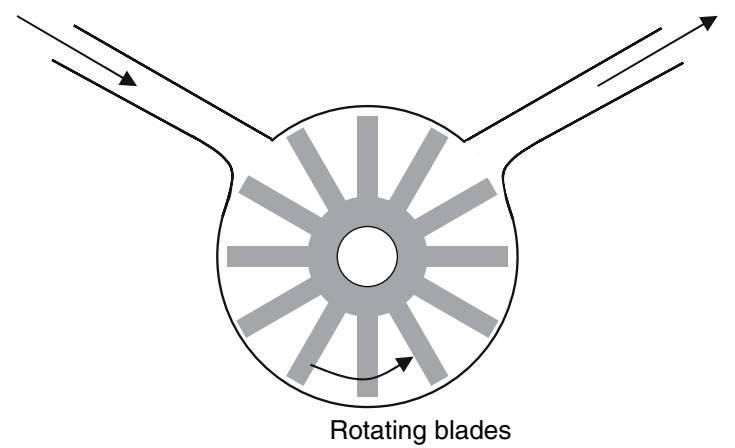

(c)

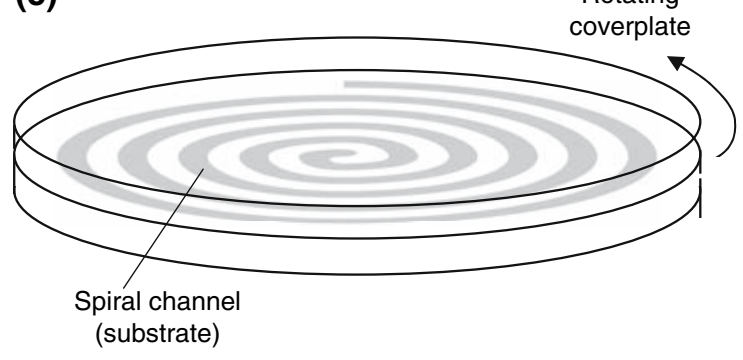

(b)

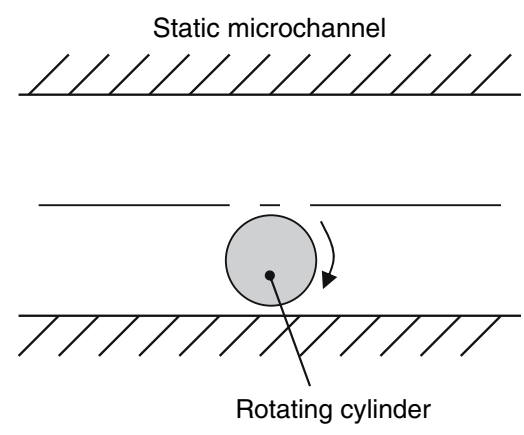

(d) Static microchannelinlet/outlet

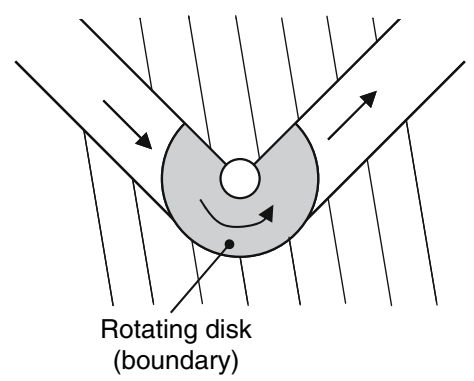

of a rotating shaft in other channel geometries (straight, Lshaped, U-shaped) has also been numerically investigated (da Silva et al. 2007).

Spiral-channel viscous pumps operate similar to Couette flow in that the movement of the boundary induces viscous stress on the fluid near the wall (Fig. 8c). However, unlike traditional Couette flow, the channel is shaped in a spiral fashion such that a rotating upper boundary can be used as opposed to linear boundary motion. Numerical studies have been conducted showing that channel aspect ratios less than 10 result in greater than $5 \%$ error when modeling using a 2D approximation (Kilani et al. 2006). Spiral curvature and stream function solutions for analyzing this type of system have also been reported (Al-Halhouli et al. 2007; Haik et al. 2007).

Disk viscous micropumps generate flow by the rotation of a disk that also acts as a boundary to the channel flow (Fig. 8d). Single- and double-disk viscous pumps are similar to Couette flow and have been studied and fabricated by Blanchard et al. (2005). The rotational movement of the $\operatorname{disk}(\mathrm{s})$ induces viscous stresses on the fluid that forces the fluid from an inlet channel, through the pumping volume above the single disk (or between the two disks) towards the outlet channel. The benefit over spiral pumps is that they are easier to fabricate; the benefit over eccentrically rotating shafts is larger flow rates and generated pressures.

\section{Electro- and magneto-kinetic micropumping techniques}

Electro- and magneto-kinetic micropumps directly convert electrical and magnetic forms of energy into fluid motion. Since these pumping processes occur in a continuous manner, the resulting flow is generally constant/ steady. Electrokinetic pumps often utilize an electric field to pull ions within the pumping channel, in turn dragging along the bulk fluid by momentum transfer due to viscosity. Magnetokinetic pumps typically utilize the Lorentz force on the bulk fluid to drive the microchannel flow. Further, dynamic pumps typically are valveless, gaining their directionality from the direction of the applied force.

\subsection{Electrohydrodynamic pumps}

Electrohydrodynamic (EHD) pumps utilize electrostatic forces acting on dielectric liquids to generate flow. There are several types of EHD pumps, and the distinction is based mainly on the method by which the charged particles are introduced into the fluid. The body force acting on the fluid resulting from the interaction of a non-homogeneous electric field $E$ with a fluid space charge density $q_{f}$ is given by the relation (Melcher 1981) 


$$
\begin{aligned}
F= & \underbrace{q_{f} E}_{\text {Coulomb Force }}+\underbrace{P \cdot \nabla E}_{\text {Polarization Force }}-\underbrace{\frac{1}{2} E^{2} \nabla \varepsilon}_{\text {Dielectric Force }} \\
& +\underbrace{\nabla\left(\frac{1}{2} \rho \frac{\partial \varepsilon}{\partial \rho} E^{2}\right)}_{\text {Electrostrictive Force }}
\end{aligned}
$$

where $P$ is the polarization vector, $\varepsilon$ the fluid permittivity and $\rho$ the fluid density.

\subsubsection{Induction-type EHD}

Induction-type EHD pumps require either a gradient in the electrical conductivity or permittivity of the working fluid. This is typically achieved by anisotropic fluid heating or by discontinuities in properties, which occurs for layers of non-mixing fluids or suspended particles in the fluid. Alternating voltages are imposed on the electrodes present on the boundary of the fluid channel. These voltages vary in time, creating a traveling wave that moves through the working fluid, perpendicular to the gradient in conductivity (Fig. 9a). Charges are induced by the traveling electric field waves at the interfaces of the media, or in the bulk of the working fluid where the gradients in conductivity or permittivity occur. The charges are attracted or repelled by the space- and time-varying electric field and carry with them the bulk fluid due to viscous effects. Charges neutralize in a time period on the order of the charge relaxation time. Hence, short distances between electrodes allow the charges to move from one electrode to the other before being neutralized.

The direction of motion is dependent upon the direction of the traveling wave and the temperature gradient. For attraction-type EHD the charges move in the same direction as the potential wave and maximum velocities are limited by the speed of the potential wave. For repulsiontype EHD, like charges are repelled by the electrodes away
Fig. 9 EHD micropumps with a traveling-wave, inductiontype, b planar injection-type, and $\mathbf{c}$ polarization-type driving mechanisms (a)

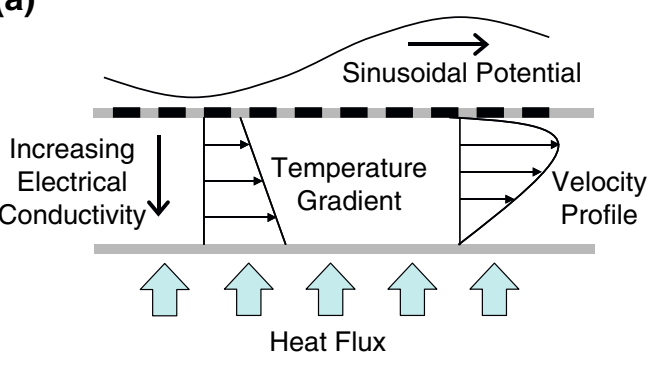

(b)

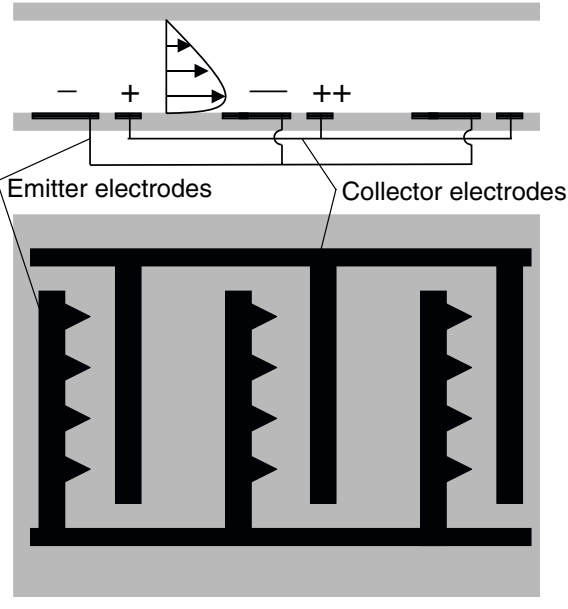

(c)

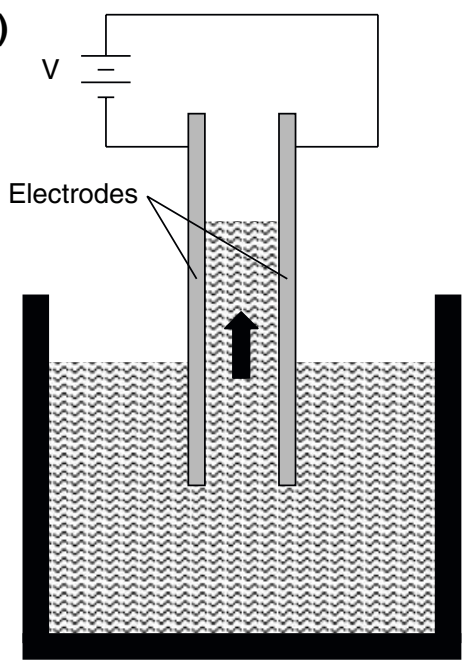


from the plate resulting in a direction opposite that of the traveling wave. In this mode, there is no traveling waveimposed limit on the speed of the flow.

Induction EHD pumps are dependent upon the electrical properties of the fluid (permittivity and conductivity). Conductivities often vary between $10^{-14}$ and $10^{-9} \mathrm{~S}$ which limits fluid selection. Non-conducting or non-ionic fluids are commonly used (Woias 2005). In the very recent past, more conductive fluids $(\sim 140 \mu \mathrm{S} / \mathrm{cm})$ have been demonstrated for use in a traveling-wave, induction EHD pump (Felten et al. 2006). They show velocities of $10-80 \mu \mathrm{m} / \mathrm{s}$ for different temperature gradients across the pump channel. For the conductive solutions used in this study, the traveling-wave frequency is very high $(3.1 \mathrm{MHz})$.

The attractiveness of induction EHD lies in part in that the pump components can be fabricated by standard surface micromachining methods directly in the channel, thus reducing overall pump size and enhancing ease of fabrication. It is this amenability to integration that makes them applicable for use in electronics packaging (Singhal and Garimella 2007) and $\mu$ TAS systems.

A hybrid pump, combining the use of both a nozzlediffuser diaphragm pump and induction EHD has been developed (Singhal and Garimella 2005b). A numerical model demonstrating its capabilities was presented using theory and experimental results in the literature for validation. This paper marks the first time the force density approach has been used to successfully model induction EHD as compared to the electric shear stress approach used earlier. An efficiency study of EHD pumping was also reported (Singhal and Garimella 2005a).

\subsubsection{Injection-type EHD}

In injection-type EHD micropumps, electrochemical reactions at the electrodes cause the injection of free ions into the bulk liquid. These ions experience Coulomb forces due to the presence of the electric field. This causes the movement of ions, which in turn carry the bulk fluid with them. A basic, injection-type EHD pump is illustrated in Fig. 9b. EHD pumps, in general, work for low-conductivity fluids such as some organic fluids, and non-polar fluids (e.g., alcohols), while induction-type pumps can operate with higher conductivity fluids. De-ionized water can also be pumped by injection-type pumps, although not very efficiently because of the resulting electrolysis.

A planar, injection EHD pump has been developed utilizing sawtooth electrodes on the boundary (Darabi and Wang 2005). The target application of cryogenics used liquids such as HFE-7100 and liquid nitrogen. Specifically, this work demonstrated the feasibility of injection EHD in cryogenics.
A similar injection-type pump capitalizing on Corona discharge for injection of ions into air has also been used to pump air flows with potential applications in the electronics cooling industry (Go et al. 2007).

\subsubsection{Polarization-type EHD}

A polarization-type EHD micropump uses a non-homogeneous electric field through the working fluid to create a variation in the fluid electric field density. A gradient in the energy of the dipoles (from the dipolar liquid) is generated. Dipoles in regions of high electric field have lower energy. Therefore, dipoles in the section of channel bounded by the electrodes will have lower energies than those outside the electrode region and fluid external to the electrode section will move into the channel causing a pumping action (Fig. 9c). This mechanism has appeared most recently in thin-film evaporator applications (Moghaddam and Ohadi 2005) and 3D micropump modeling (Lin and Jang 2005).

\subsubsection{Ion-drag}

Most electrokinetic pumping mechanisms (such as induction EHD, injection EHD, electroosmosis, etc.) use ion drag as the force to drive the fluid. However, most of the micropumps in the literature referred to as being ion-drag pumps are injection-type EHD pumps (Darabi et al. 2002; Seyed-Yagoobi 2005).

\subsection{Electroosmotic pumps}

Electroosmotic (EO) pumping exploits the surface charge that can spontaneously develop when a liquid comes in contact with a channel wall or that can be artificially created using electrodes. Oppositely charged ions in the fluid shield the surface charge and can be manipulated with a DC or AC electric field. ACEO flow is a developing area of research; both ACEO and DCEO flow has received much attention in recent years.

\subsubsection{DC electroosmotic}

DC electroosmotic pumps can be constructed using fused silica or glass capillaries with electrodes that provide an electric field along the channel length (see Fig. 10). For silica-based channels, when an electrolytic solution (with $\mathrm{pH}$ greater than $\sim 4$ ) comes in contact with the channel wall, the surface silanol groups spontaneously deprotonate, leaving a negatively charged boundary. The induced 


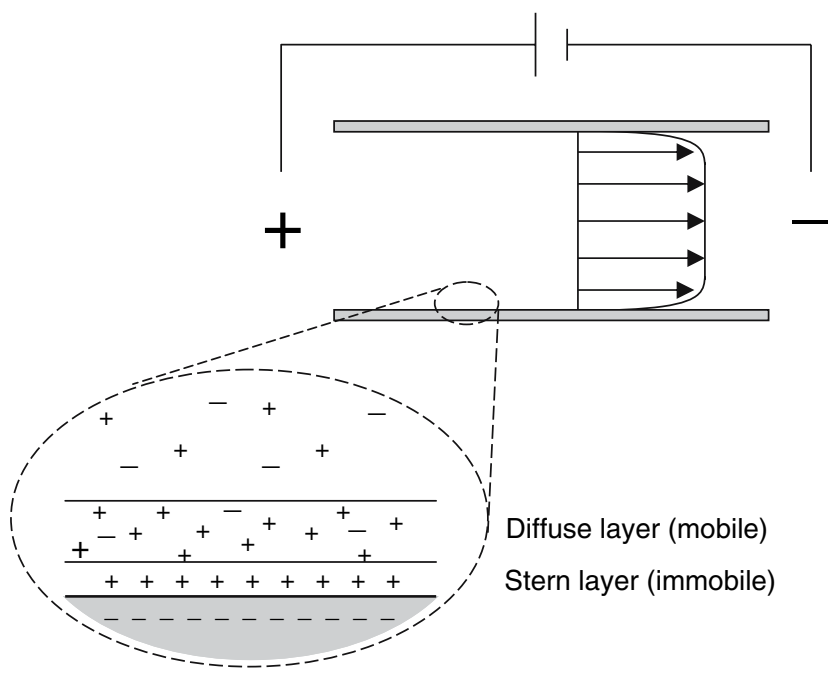

Fig. 10 DC electroosmotic micropump and corresponding flow profile

surface charge attracts positively charged ions and repels negatively charged ions in the solution, resulting in the formation of an electric double layer along the capillary wall and a neutral charge density in the center of the channel. The electric double layer thickness can be calculated by (Chen et al. 2005b)

$\lambda_{D}=\sqrt{\frac{\varepsilon \kappa T}{2 q^{2} z^{2} c}}$

where $\varepsilon$ and $T$ are the permittivity and temperature of the fluid, $z$ and $c$ are the valence number and average molar ion concentration, $\kappa$ is the Boltzmann constant and $q$ is the charge of an electron. With an applied DC electric field, the force experienced by the fluid near the capillary wall is much higher due to the high-charge density in its vicinity. These charges move in response to the electric field and the fluid motion is propagated to the channel interior due to viscous forces. When the channel hydraulic diameter is much larger than the Debye length, the velocity profile is nearly uniform across the cross-section, and the resulting velocity can be described by the Helmholtz-Smoluchowski equation (Iverson et al. 2004)

$u=-\frac{\varepsilon \zeta}{\mu} \frac{d V}{d x}$

where $\zeta$ is the zeta potential at the wall and $d V / d x$ is the voltage gradient (electric field). For channel widths small enough that the electric double layers overlap $(<1 \mu \mathrm{m})$, the commonly used Poisson-Boltzmann equation does not represent EO pumping well. Models aimed at this operating region have been developed ( $\mathrm{Hu}$ and Chao 2007).

There are two main challenges for electroosmotic pumps. First, bubble generation can occur from the large currents in the open channel. Electrolysis and reactions at the electrodes take place and produce ions that can contaminate the sample and generate bubbles, which can block microchannels. Typically, a bubble-release mechanism is incorporated downstream or the current is reduced by using dense particle packing within the channel. Second, electroosmotic pumps with an open channel typically have low stall pressures. High pressure build-up can be achieved by using very small channels or if the channel is densely packed. Thus some of the disadvantages of electroosmotic pumps are often alleviated by controlling particle packing in the channel. Some designs have used small packed particles; others have used monolithic materials to achieve the dense channel packing. Chen et al. (2005b) developed a monolithic silica matrix EO pump using a sol-gel process and achieved pressures as high as $400 \mathrm{kPa}$.

Several papers incorporating packed beads have been presented in recent years (Kang et al. 2007; Piyasena et al. 2006; Zeng et al. 2001). The packed beads increase the interfacial area available for EO flow, thereby increasing maximum pressure; pressures above $2 \mathrm{MPa}$ have been reported (Zeng et al. 2001). As an alternative to the use of packed beads, an innovative EO pump method using porous anodic alumina (PAA) was reported (Vajandar et al. 2007). This pump had the highest normalized flow rate as compared to any previous EO pump at lower voltages. The PAA was coated with a sol-gel $\mathrm{SiO}_{2}$ in order to obtain a high zeta potential.

Other methods using EO pumps in series or parallel have demonstrated improvement over traditional singlestage pumps. A multi-stage EO pump constructed using several porous silica-packed pumping segments in series was tested with the same driving voltage applied across each of the segments to achieve pressures as high as $10 \mathrm{MPa}$ (Chen et al. 2005a). While the flow rate was not shown to increase for the pumps in series, the maximumback pressure increased roughly in proportion to the number of segments. Therefore, the maximum-back pressure attained is increased without an increase in the driving voltage; however, the power consumption does increase due to the higher current draw. For a single-stage pump a much higher voltage is necessary to generate the same pressure.

Non-polar liquids with low conductivity $\left(<10^{-6} \mathrm{~S} / \mathrm{m}\right)$ do not form the necessary double layer for EO pumping to occur. To pump such liquids with EO flow, a two-liquid viscous EO pump was designed by Brask et al. (2005); the design used a thin layer of conducting pumping liquid driven by electro-osmosis to drag a non-conducting working liquid by viscous forces. In effect, the conducting liquid forms a sheath, providing a Couette flow-like moving boundary to the non-conducting liquid.

For those applications in which heat transfer between fluid and substrate is important, thermally developing, EO 
flow studies including both cylindrical (Broderick et al. 2005) and rectangular (Iverson et al. 2004) microchannel geometries have been conducted.

\subsubsection{AC electroosmotic}

AC electroosmotic flow has emerged as a viable microscale pumping mechanism for conductive or electrolytic solutions. Unlike the deprotonation on the channel surface for DCEO flow, electrodes positioned on the channel boundary provide the charge to establish the electric double layer in this case. The most common geometry is to use asymmetric electrodes to induce an electric field and draw the diffuse layer charges along the surface of the electrodes (see Fig. 11). The advantage of ACEO pumps is that relatively high velocities can be achieved for very small voltages of less than $10 \mathrm{~V}$ (Debesset et al. 2004; Mpholo et al. 2003). Further, as voltages are increased within this range, flow reversal can be achieved making a pump of this type bi-directional. In fact, reverse flow velocities are found to be larger than forward flow velocities (Garcia-Sanchez et al. 2006; Vijendran et al. 2006). A physical explanation of the observed phenomena for several experimental studies involving ACEO was offered by Olesen et al. (2006). Most ACEO pump studies have reported only measured velocities (up to $\sim 500 \mu \mathrm{m}$ / s) for recirculating flow with little information provided regarding pressure.

A few variations on the basic ACEO device have been considered. Three-dimensional electrodes have been investigated and shown to increase fluid velocities over a corresponding planar geometry. A second, asymmetric metal layer deposited on top of a symmetric array of electrodes demonstrated an order-of-magnitude increase in velocity (Urbanski et al. 2006). Traveling-wave (GarciaSanchez et al. 2006) and biased (Wu 2006) ACEO flow have also been studied with possible use in the transport and concentration of particles for biological applications.

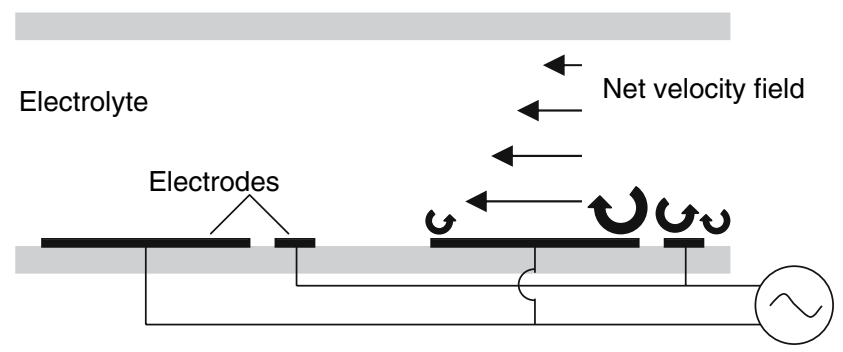

Fig. 11 AC electroosmotic flow and corresponding flow profile

\subsection{Magnetohydrodynamic pumps}

Magnetohydrodynamic (MHD) pumps exploit the Lorentz force that is generated when a current-carrying conductor is placed in a magnetic field. The resulting direction of the force is perpendicular to both the magnetic and electric fields. Figure 12 illustrates the basic design of an MHD pump. An electric field is generated by electrodes on opposite walls of the channel. A magnetic field (perpendicular to the electric field) is established using permanent magnets (or electromagnets) on opposite sides of the remaining two walls of the channel. As a result, the fluid experiences a Lorentz force acting along the length of the channel, which leads to induced flow. The Lorentz force in a rectangular channel where $I$ is the current across the fluid between the channel electrodes, $w$ is the distance between electrodes and $B$ is the magnetic field strength can be calculated by

$\vec{F}=\vec{I} \times \vec{B} w$.

Or, using the current density $J$, we can arrive at the maximum pressure over an electrode region of length, $l$,

$\vec{P}_{\max }=\vec{J} \times \vec{B} l$.

For laminar flow, the maximum flow rate can be obtained by (Laser and Santiago 2004)

$\vec{Q}_{\max }=(\vec{J} \times \vec{B}) \frac{\pi D_{\mathrm{h}}^{2}}{128 \mu}$

where $D_{\mathrm{h}}$ is the hydraulic diameter and $\mu$ is the fluid viscosity. The working fluid must be conductive for MHD operation; electrolytic solutions are most commonly used. One major challenge of MHD pumps is Joule heating from the electrical current in the fluid (Duwairi and Abdullah 2007; Patel and Kassegne 2007).

Several MHD pumps in the literature were compared against a recent MHD pump designed for use in a nuclear magnetic resonance (NMR) environment by Homsy et al. (2007). In general, the best flow rate performance is

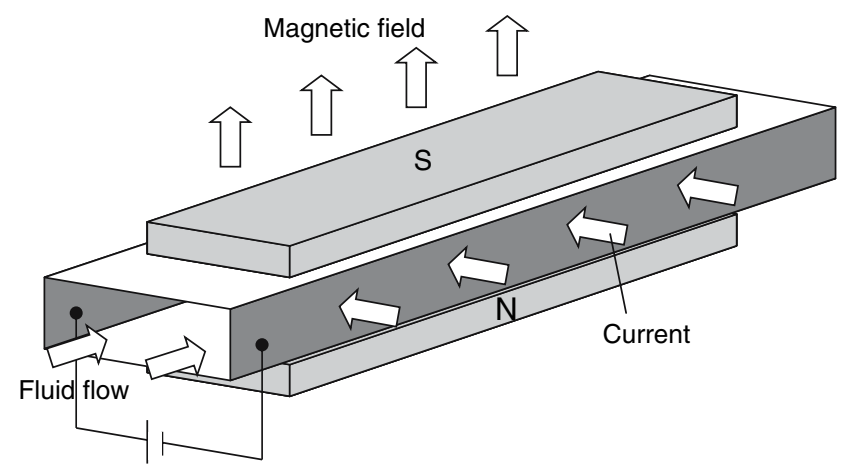

Fig. 12 Magnetohydrodynamic micropump 
obtained for maximum current density, magnetic field intensity, and channel cross-sectional area. MHD pumps with the highest body force are obtained when the current density, magnetic field intensity and electrode length are maximized (by minimizing the cross-sectional area). In an NMR environment, this pump by Homsy et al. (2007) was shown to perform the best as compared to other MHD pumps under such operating conditions but the superconductive magnet used was several feet high.

\subsection{Electrowetting pumps}

Liquid metals in contact with electrolytic solutions develop charged interfaces that act like a capacitor due to electrochemical reactions. When an electric voltage is applied along the interface of a liquid metal droplet in an electrolyte, charge redistribution occurs resulting in a gradient in surface tension at the interface, which causes movement of the droplet to regions of lower surface tension. Switching the direction of the applied voltage also changes the direction of motion. This switching of droplet motion has been used in the past to actuate a vibrating diaphragm by creating a pressure gradient in the electrolyte solution to deflect the diaphragm (Yun et al. 2002).

However, increased interest has arisen for incorporating electrowetting-based fluid delivery/manipulation techniques into lab-on-a-chip applications. The principle is to use an array of electrodes that could be individually controlled for moving droplets in any planar direction on a surface such that they could be introduced to other droplets for mixing and/or chemical reactions. A recent topical review of electrowetting includes a discussion of the challenges of this work and reviews the state of the art (Mugele and Baret 2005). Energy-based models of droplet manipulation with electrowetting on flat and rough surfaces have also been developed (Bahadur and Garimella 2006, 2007).

\subsection{Other}

Other interesting micropumping techniques include optoelectrostatic microvortex and flexural plate wave driving mechanisms.

\subsubsection{Optoelectrostatic microvortex}

Fluid flows have been generated with an optoelectrostatic microvortex (OEMV) mechanism in which a vortex-like fluid flow is generated around the focal point of a laser beam in the presence of an intense AC electric field (Nakano et al. 2007). The focal point of an 1064-nm IR laser beam in close proximity to an electrode producing an AC electric field generates flow toward a counter electrode with velocities of hundreds of micrometers per second (Fig. 13). The flow velocity was significantly affected by the position of the IR laser beam focal point in the $z$ direction (out of the page), with higher velocities achieved with increasing distance from the electrode substrate.

\subsubsection{Flexural plate wave pumps}

A flexural plate wave micropump consists of an array of thin piezoelectric material strips deposited on a relatively thin substrate (schematic shown in Fig. 14). Alternating piezoelectric strips are connected to two different electrodes and are actuated at very high frequencies. A flexural wave is established in the composite membrane, resulting in the formation of a high-intensity acoustic field near the membrane surface. The acoustic field leads to fluid motion near the membrane surface in the direction of the wave. This topic has not been further developed in recent years (Luginbuhl et al. 1998; Nguyen et al. 2000).

\section{Evaluation and discussion of micropumping technologies}

As described above and in previous micropump reviews, a wide variety of possible pumping mechanisms have been proposed for the emerging needs in microscale flows. As pump sizes have reduced to the microdomain, the effect of centrifugal and inertial forces are generally limited. The large surface-to-volume ratios instead amplify the effects of viscous forces, often making them the dominant force. Additionally, the force per unit volume of some pumping techniques actually increases with reduction in length scale. In this section, the various pumping technologies included in this review are compared qualitatively and

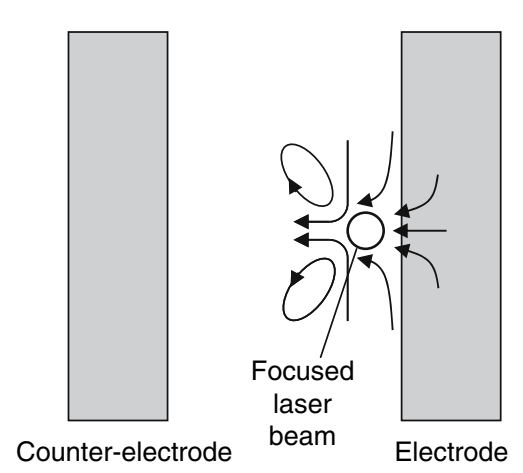

Fig. 13 Optoelectrostatic microvortex (OEMV) driving mechanism for micropumping 


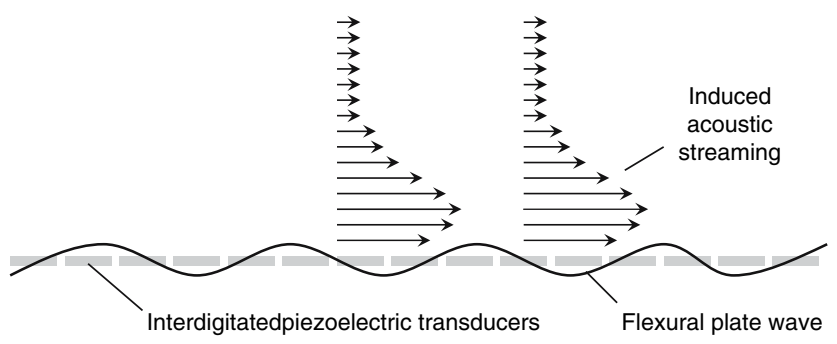

Fig. 14 Flexural plate wave micropump

quantitatively, especially with respect to a few specific applications.

\subsection{Application-driven requirements}

Determination of pumping requirements and environment is the first step in selecting a micropumping technique for a given application. A few specific applications are listed below. The references included indicate the types of pumps that have been considered for these applications, but it is not implied that these are the best pumping solutions.

\subsubsection{Controlled insulin delivery pumps}

Insulin delivery devices generally do not require high flow rates. Rather, the critical features of pumps in this application is that they provide precisely-metered, small doses and that the flow is able to operate independent of back pressure (Geipel et al. 2007). Thus, constant flow over a range of environmental-back pressures drives the design and valving.

\subsubsection{Thermal management of micro devices}

Since liquid flow is used in this application as a heat transport mechanism, large flow rates are normally called for to accommodate the high heat fluxes. Since fins or microchannels are used to increase the surface area available for heat transfer, these devices also demand the capability of overcoming high-pressure drops. Developing micropumps that simultaneously deliver on both of these attributes is challenging. Also, pumps used in this application are expected to last the lifetime of the electronic device as opposed to the disposable micropumps common in biological applications. Piezoelectric pumps have been used in thermal management solutions owing to their low power consumption (Faulkner et al. 2006). Further, integration of micropumps with the packaging holds particular promise as it reduces the thermal interface resistances inherent at mechanical joints (Garimella and Singhal 2004; Garimella et al. 2006). Self-cooled printed circuit boards (PCB) have also been conceived of wherein the pump and microfluidics are integrated into the PCB (Nguyen and Huang 2005).

\subsubsection{Chemical and biological analysis pumps}

Micropumps for these applications can have a wide range of desired flow rates and pressure drops. However, the reduction of sample or reagent quantities is almost universally desirable. Hence, precision control of small volumes is of particular importance. User intervention is to be avoided to prevent contamination. Likewise, containment of chemical and biological agents can be imperative as they are often hazardous. Further, the devices should be low-cost and disposable to avoid contamination effects. Pneumatic pumps have been used for cell culturing (Huang and Lee 2007) and surface plasmon resonance (SPR) detection (Huang et al. 2006b). Electrochemical pumps have appeared for use in influenza A subtype identification and sequencing (Liu et al. 2006a) and in DNA microarray processing (Liu et al. 2006b).

\subsubsection{Cell sorting and cytometry}

By and large, all of the points addressed above in the chemical and biological analysis pumps apply with some possible additional restrictions in this application. Often, fluid sheathing is used as a technique for isolation of the sample liquid. Sheathing can be maintained when the flow is laminar and the streamlines do not cross. Thus, diaphragm pumps, or those that have the potential to induce oscillatory, turbulent or otherwise chaotic flows would typically need to be avoided. For large throughputs, high flow rates would also be needed; however, the current limitation appears to be the sorting technique as opposed to pumping of the fluid. As an example, s-shaped peristaltic pumps have found application in cell sorting and cytometry (Yang et al. 2006).

\subsubsection{PCR pumps}

As with the chemical and biological analysis tools, pumps for PCR are commonly designed to be disposable. Thus materials and manufacturing are important considerations. Electrokinetic pumping has been commonly used due to device simplicity. However, although electroosmotic pumping has been used for this purpose, the flat velocity profile reduces sample dispersion. Further, separation of 
components in a heterogeneous mixture due to different electrophoretic mobilities (electrophoretic demixing) is unfavorable in PCR assays (Zhang et al. 2007). Pneumatic and other diaphragm pumps have not been commonly used in this application due to device complexity and larger pump footprints.

\subsubsection{Fuel cells}

An efficient fuel delivery system is required to simultaneously provide an adequate amount of fuel for consumption and remove carbon dioxide from the fuel cell device. Direct methanol fuel cells have been considered for portable electronic applications. Micropumps for this application are specifically required to have low energy consumption, while maintaining sufficient flow rates for fuel delivery (Zhang and Wang 2005).

\subsubsection{DNA hybridization}

A limiting factor in DNA research is the long hybridization times required for strong detection signals. Spatial variations in binding can also occur due to the large diffusion lengths involved. Small molecules can diffuse much more easily than larger protein molecules, thus increasing the hybridization times for larger molecules. When the hybridization is static, only very small percentages of the reagent are actually introduced to the probes on the chip. Mixing can significantly reduce binding times as well as increase the amount of reagent presented to the probes (Vijendran et al. 2006). The bi-directional flow capability of $\mathrm{AC}$ electroosmosis, peristaltic and other pumps would allow DNA or biological materials in solution to be driven back and forth over probes or measurement regions.

\subsection{Materials and construction}

Materials selection plays an important role beyond cost in chemical, biological and electrical systems. Compatibility with environmental and operating conditions is imperative for proper device functioning. Construction using these materials is also a concern when considering the environment, chemicals or stress to which a device may be subjected. Table 1 lists the Young's Modulus and linear coefficient of thermal expansion (CTE) for a variety of commonly used materials found in micropump devices. Depending upon the application, materials selection may drive the construction method, or vice versa.

Diaphragm material selection needs to account for the frequency of vibration, which is often dependent on the actuation method. For low-frequency diaphragm pumps, which provide a low force, a low-modulus diaphragm material provides the maximum change in volume. For high-frequency driving mechanisms, good performance is obtained using stiff diaphragms, which provide ample force and a fast mechanical response.

Diaphragm displacement pumps in the literature have often capitalized on flexible membranes to provide reliable pumping. Flexible diaphragms with large deflection amplitudes are often self-priming and bubble-tolerant. Parylene and PDMS are becoming more common as diaphragm materials in micropumps as they are more flexible than the traditional silicon or nitride diaphragms and are also more bio-compatible. However, adhesion between the
Table 1 Young's modulus and coefficient of thermal expansion (CTE) for common micropump materials

\begin{tabular}{lll}
\hline Material & $\begin{array}{l}\text { Young's modulus } \\
(\mathrm{Gpa})\end{array}$ & $\begin{array}{l}\text { Linear coefficient of thermal } \\
\text { expansion }(\mathrm{CTE})\left(\mathrm{ppm} /{ }^{\circ} \mathrm{C}\right)\end{array}$ \\
\hline Aluminum & $47-74$ (MEMSnet 2007) & 25 (Kovacs 1998) \\
Glass & $40-80$ (MEMSnet 2007) & $0.1-3$ (Chen et al. 2000) \\
Gold & 80 (MEMSnet 2007) & 14.2 (Kovacs 1998) \\
Parylene & $2-3$ (SCS Coatings 2007) & $35-80$ (SCS Coatings 2007) \\
PDMS & $0.36-0.87$ (Armani et al. 1999) & $270-310$ (Grzybowski et al. 1999) \\
Platinum & 170 (MEMSnet 2007) & 8.8 (Kovacs 1998) \\
Polyimide & $3-15$ (MEMSnet 2007) & $3-191$ (Kovacs 1998) \\
Silicon & $125-202$ (MEMSnet 2007) & 2.6 (Kovacs 1998) \\
Silicon Dioxide & $46-92$ (MEMSnet 2007) & $0.35-0.4$ (Kovacs 1998) \\
Silicon Nitride & $100-325$ (MEMSnet 2007) & 1.6 (Kovacs 1998) \\
Silicone Rubber & 0.0062 (SCS Coatings 2007) & $250-300$ (SCS Coatings 2007) \\
Silver & 76 (MatWeb 2007) & 18.9 (Kovacs 1998) \\
Stainless Steel & $200-240$ (MEMSnet 2007) & $10-20$ (Ilavsky and Berndt 1998) \\
\hline
\end{tabular}


diaphragm membrane and chamber wall (stiction) can occur. PDMS working surfaces have been treated with a $0.1 \mathrm{M} \mathrm{NaOH}$ solution or by an $\mathrm{O}_{2}$ plasma etch to render the PDMS surface hydrophilic. This helps to reduce stiction and prevent bubble formation (Goulpeau et al. 2005). PDMS is biocompatible with many processes, namely, PCR, cell sorting and bacterial analysis, among others. PDMS has been considered as one of the platforms for a lab on a chip because it has high gas permeability (and hence, is not hermetic), low loss tangent, low chemical reactivity, optical transparency, good thermal stability, and low interfacial free energy.

Glass materials for pump body, valves and even diaphragms can be attractive in that they are chemically inert and can be sterilized at high temperatures which is especially important for lab-on-a-chip applications (Yamahata et al. 2005b).

In environments where there is thermal cycling over a range of temperatures, material expansion and contraction can cause device failure or leakage. Specifically, materials are characterized by a coefficient of thermal expansion (CTE) that quantifies the rate of volume expansion as a function of temperature (see Table 1). When two mating parts of different materials are joined at one temperature and the device temperature is increased or decreased, their volume may change at a different rate as the temperature changes. Thus, the stress induced from the volume expansion mismatch at the interface of these materials can be large enough that they disjoin, causing failure.

There are a number of bonding or joining methods that can be used for device assembly. Some involve the addition of a bonding material while others capitalize on surface chemistry and ion diffusion. Some bonding methods are able to provide both a liquid and hermetic (airtight) seal. Hermeticity can be a highly desired attribute but is not achievable by all bonding methods.

Common thin-film bonding materials consist of epoxy, polymers, parylene, polyimide, benzocyclobutane (BCB) (Hwang et al. 2004; Oberhammer et al. 2003), and glass frit, among others. The attraction of these materials is that they are conformal to uneven or wavy geometries and can fill gaps at contact planes. Typically, these materials are deposited or spun on, the two mating surfaces are brought together and then the bond is cured with either elevated temperatures or simply by exposure to air. Polymers, parylene, polyimide and BCB can all be patterned using photoresists and etching; some are even photodefinable. This offers greater control in the placement of bonding layers. Glass frit layers are commonly screen-printed and are generally comparatively thick. They also can provide hermetic seals whereas most thin-film bonding materials do not. However, glass frit materials can have lead that leaks into the working fluid.
Self-assembly using acrylate-based adhesives has been demonstrated for bonding. The acrylate-based liquid is deposited on a substrate with hydrophilic binding sites. Corresponding hydrophilic areas on the mating surface self-align with the binding sites by minimizing interfacial energies from the adhesive droplets. This method has been used to align PZT actuators that are secured in place when the adhesive is polymerized (Fang et al. 2006). The process is repeatable and has demonstrated uniformity across many pumps.

Usage of PDMS has increased dramatically as a tool for rapid prototyping (Duffy et al. 1998). As mentioned above, it is compatible with a number of chemical and biological processes, but it also can be used for bonding. An $\mathrm{O}_{2}$ plasma etch of PDMS surfaces converts the surface $\mathrm{O}-$ $\mathrm{Si}\left(\mathrm{CH}_{3}\right)_{2}$ group into a silanol $(\mathrm{OH})$ group which, in turn, changes the surface chemistry from hydrophobic to hydrophilic (Tang et al. 2006). This improves adhesiveness of the PDMS surface for bonding to other substrates without intermediary layers. The simplicity of casting and bonding of PDMS has led to its pervasive usage.

Anodic bonding is commonly performed between glass (Pyrex) and silicon substrates to create a hermetic seal (Cozma and Puers 1995; Wallis and Pomerantz 1969). The parts to be joined are heated $\left(\sim 400^{\circ} \mathrm{C}\right)$ and clamped between metal electrodes. The temperature increase causes alkali oxides in the glass to dissociate into positive and negative ions. A high DC potential (up to $\sim 1 \mathrm{kV}$ ) used to generate an electric field across the electrodes depletes the positive sodium in the glass from the interface. The high electrostatic forces thus developed at the interface move the negative oxygen ions towards the silicon where they oxidize and create strong silicon-oxygen bonds. The challenge with this method is that the mating surfaces must be very flat since the migration of the ions occurs over a very short distance. Hence, bonding over wavy surfaces (common with electrical interconnects into the pump interior) is ineffective without planarization.

A recently introduced method of ultrasonic welding has also been demonstrated to bond inert polymers such as PEEK and PMMA (Truckenmuller et al. 2006).

\subsection{Micropump selection guidelines}

Micropumping techniques with corresponding selection guidelines are compared in this section. A quantitative comparison of various micropumps is presented in Table 2. The pumps are compared based on their maximum reported flow rate per unit planform area, maximum achievable back pressure, voltage, power input, and frequency of operation for peak performance. In general, since the flow rate in a micropump will increase with increasing size, the 


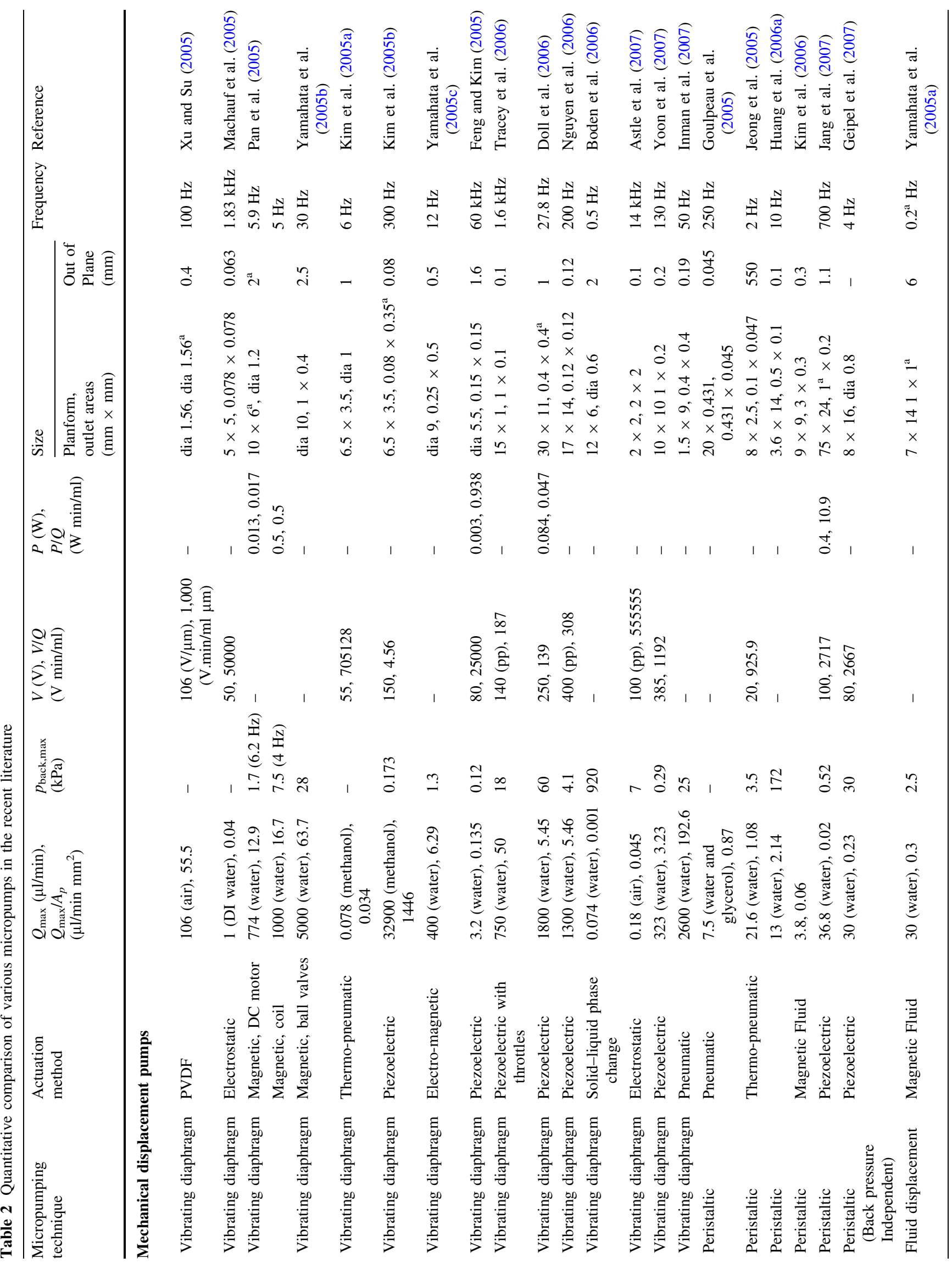




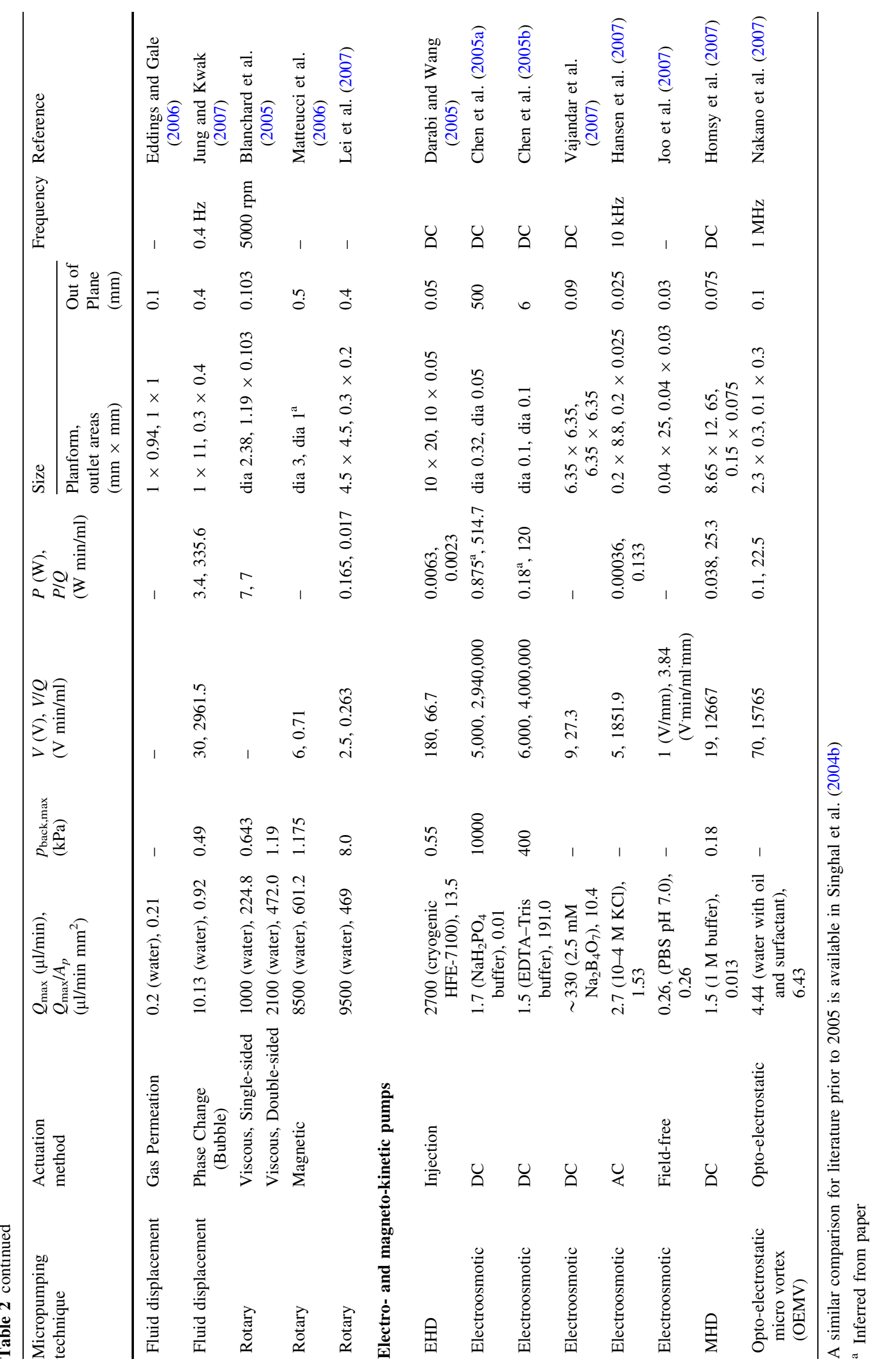


flow rate per unit planform area is calculated and tabulated. We designate the planform area as being the area corresponding to the largest cross-section of the pump and its components (usually "top-down"). For vibrating diaphragm micropumps, the area perpendicular to the primary direction of diaphragm deflection is used for determining flow rate per unit planform area. For EHD, MHD and electroosmotic pumps, the area is typically determined using the microchannel width and length. However, the micropump outlet area is also tabulated in Table 2 for reference. This outlet area corresponds to the cross-sectional area of the outlet which connects the pump (and all its components, valves, etc.) to external plumbing. Since the plumbing may always be reduced in size we have excluded it from consideration. In many cases, the outlet area corresponds to (or is on the order of) the minimum cross-sectional area of the pump.

Since there is little ambiguity in maximum reported back pressures, this metric provides an excellent comparison across all types of pumping techniques. Figure 15a illustrates this comparison for pumps included in Table 2. Not all investigations have reported maximum back pressure, especially those dealing with emerging pumping technologies such as AC electroosmotic flow, among others. As indicated in Fig. 15a, DC electroosmotic pumps generate consistently high-back pressures with a very simple geometry. Further, vibrating diaphragm pumps report a wide range of back pressures; a thermally actuated, solid-liquid phase change paraffin used by Boden et al. (2006) demonstrates the highest back pressure among the diaphragm pumps included in this comparison. Piezoelectric actuation can provide a range of back pressures, with several providing values above $10 \mathrm{kPa}$. Figure $15 \mathrm{~b}$ indicates that the piezoelectric diaphragm, rotary-disk viscous, EHD, and EO pumps have the highest flow rates per unit planform area of the pumps listed in Table 2. This scaled flow rate quantifies the amount of flow per unit of actual "real estate" required to achieve that flow, thereby eliminating the effect of overall pump size. Figure $15 \mathrm{c}$ illustrates the flow rate per unit outlet area for the same pumps included in Fig. 15b. Scaling the flow rate in this manner gives a relative comparison of output velocity, important for viscous dissipation and transit time through the flow network.

\subsubsection{Mechanical displacement pump guidelines}

When considering reciprocating or displacement pumps that exert oscillatory or rotational pressure forces on the working fluid through moving boundaries, the following points should be carefully considered for the application.
4.3.1.1 Bubble susceptibility Microscale displacement pumps often contain a pump chamber, actuator (deformable plate, etc.) and valves (often passive). Susceptibility to bubbles can pose a significant problem for these pumps and/or valving. Peristaltic pumps can be bubble-tolerant and capable of self-priming and providing bi-directional flows (Jang et al. 2007).

4.3.1.2 Bi-directional capability Depending on the application, flow reversal may be important to device operation. Peristaltic pumps are capable of providing bidirectional flows since diaphragm actuation provides the valving, whereas for nozzle/diffuser or fixed-geometry, flap valves, the flow is only uni-directional (Jang et al. 2007). However, bi-directional flow has been achieved by incorporating more than one pump into the design and alternating the actuation of the pumps (Yoon et al. 2007).

4.3.1.3 Scaling Reduction of pump diaphragm diameter adversely affects pump performance. More specifically, maximum pressure reduces with diaphragm diameter. If selecting a vibrating diaphragm pump where large-back pressures are required and space is highly constrained, this may not be the best solution.

4.3.1.4 Valving Although a discussion of valve types was provided in Sect. 2.1.9, it is noted here that displacement diaphragm pumps (or pumps with a reciprocating motion) require some sort of valving. Generally speaking, dynamic pumps do not require valves since their flow directionality is achieved by the application of a driving potential. Fixedgeometry valves are attractive from the point of view that they require no moving parts, but they do not always provide the highest back pressures.

4.3.1.5 Constant or pulsatile flow With diaphragm pumps, the flow output would generally be pulsatile. However, some researchers have developed fluid capacitors to mitigate the effects of the reciprocating motion on the flow to render it steady (Inman et al. 2007).

4.3.1.6 Actuation mechanism Selection of the driving mechanism significantly affects the operating conditions of the pump. Key points for the most common diaphragm actuation mechanisms are summarized below

- Piezoelectric-One of the earliest mechanisms to be used in micropumps was piezoelectric actuation. It 

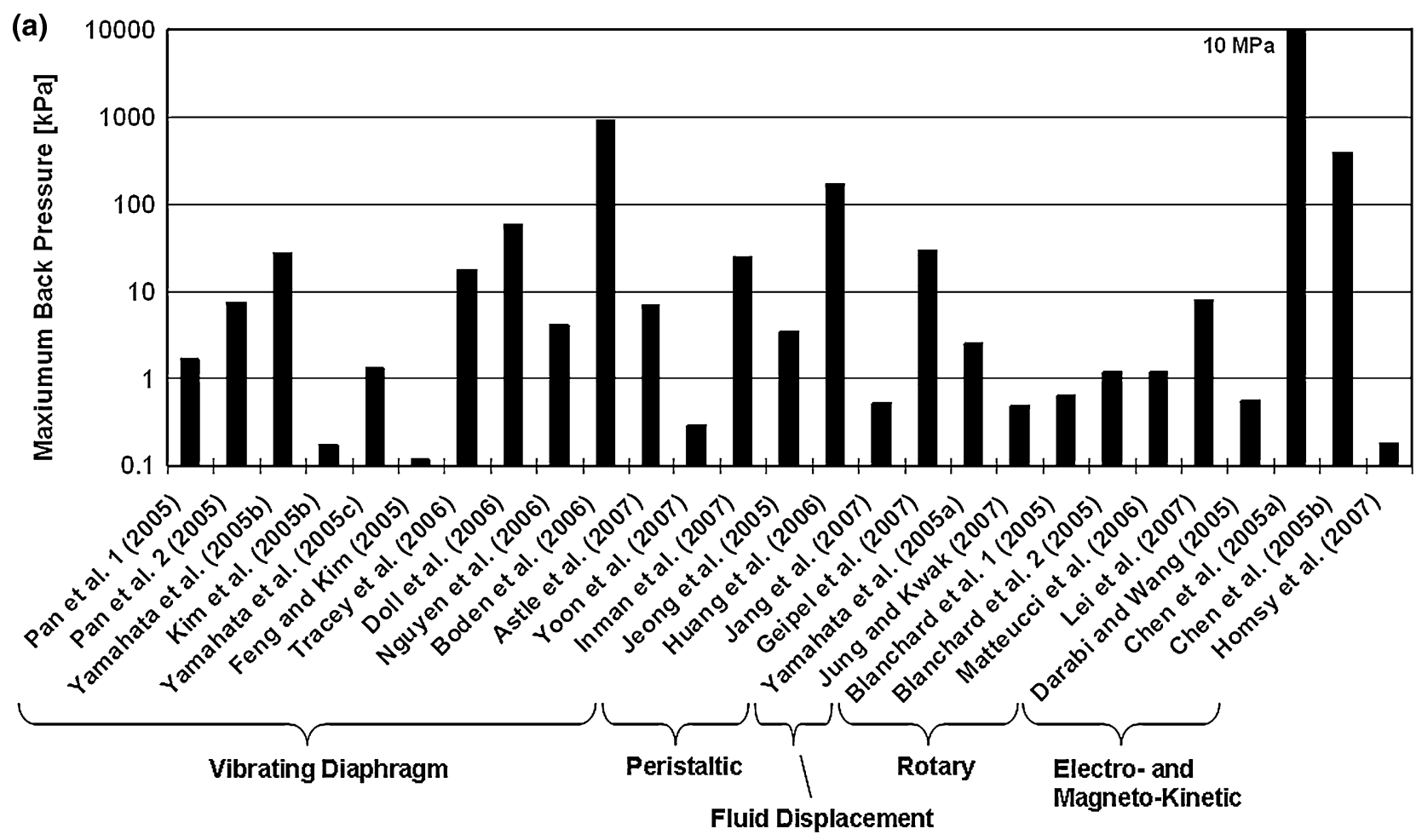

(b)
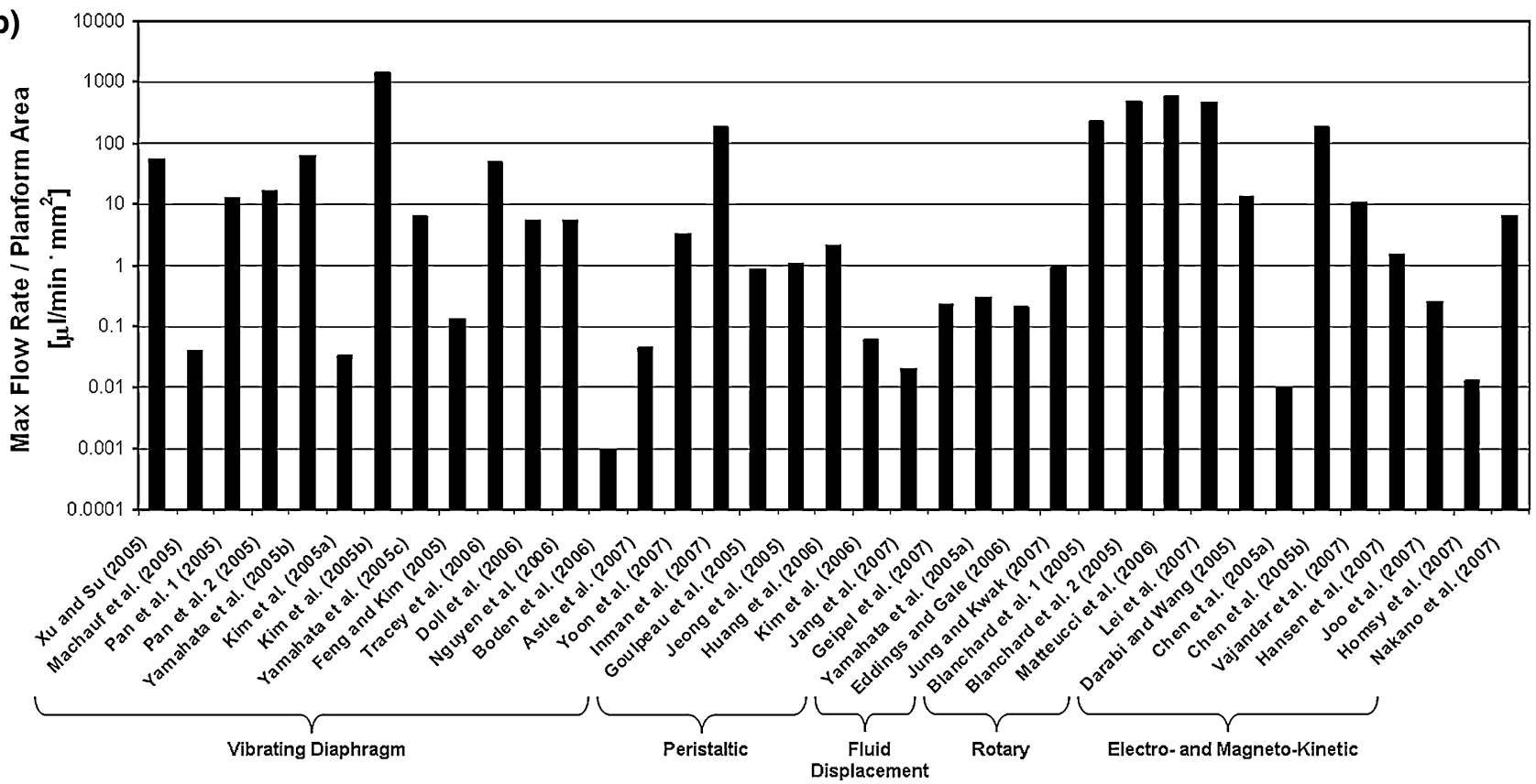

Fig. 15 Reported a maximum back pressure, $\mathbf{b}$ maximum flow rate per unit planform area and $\mathbf{c}$ maximum flow rate per unit outlet area for pumps included in Table 2

provides a high actuation force and fast mechanical response. However, relatively high actuation voltages and the attachment of PZT materials can be regarded as disadvantages. In order to address the delamination of PZT, screen-printing and thin-film deposition have emerged as alternative ways of attaching PZT. Both of these methods are advantageous from an integration standpoint but the resulting deflections are typically lower $(\sim 1 \mu \mathrm{m}$ at $100 \mathrm{~V})$ than the standard glued PZT materials $(\sim 15 \mu \mathrm{m}$ at $100 \mathrm{~V})$ (Woias 2005).

- Thermal (Thermopneumatic and Shape Memory Alloys)-These devices require low input voltages, 


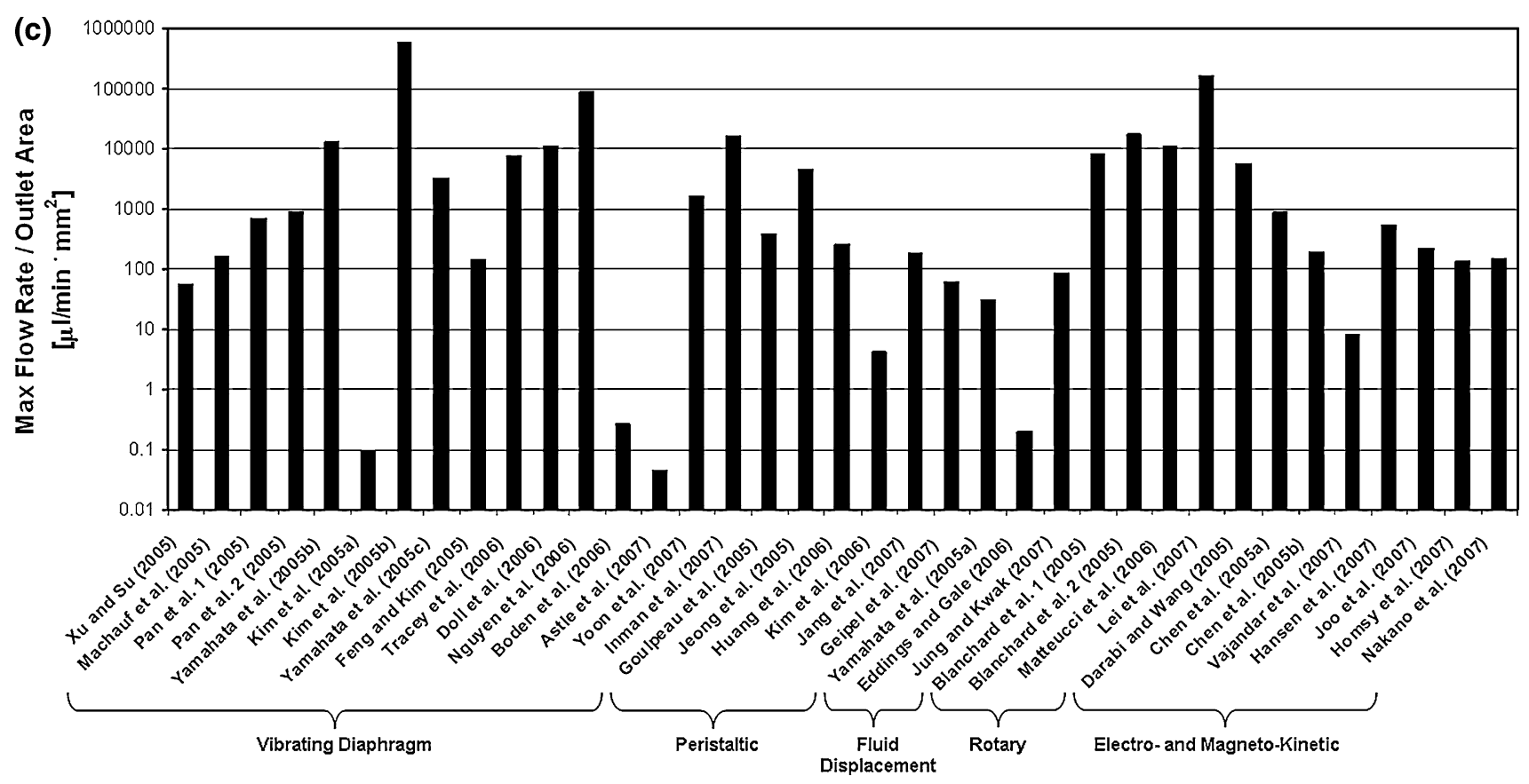

Fig. 15 continued

can generate high pumping rates and actuation forces and can be very compact. A crucial drawback of thermopneumatic actuation that has limited its use is that it has a relatively long thermal time constant, especially during the cooling process. Thus, thermopneumatic actuation frequencies are limited to about $50 \mathrm{~Hz}$ (Woias 2005). Shape memory alloys are characterized by large recoverable strain outputs up to 6$8 \%$. However, at high frequencies, shape memory alloys may not be able to cool sufficiently and their performance suffers. Generally they operate below $100 \mathrm{~Hz}$. Both mechanisms can consume a lot of power to generate heat for actuation.

- Electrostatic-Frequencies into the $\mathrm{kHz}$ range are possible with electrostatic actuation. Electrostatic devices are characterized by extremely low power consumption and full integration capability. The major disadvantages are small actuation strokes and degradation of performance with time. Also, degradation of the actuator performance has been found in some long-term high voltage operation due to the build-up of surface charges at the insulator inside the capacitor. This, in turn, reduces the internal electric field strength and corresponding stroke (Shin et al. 2005).

- Electromagnetic-This actuation mechanism is generally not as compatible with MEMS integration as the components are relatively large in comparison to others such as piezoelectric materials. Further, the electrical and mechanical properties are generally similar to thermopneumatic actuators with the advantage of a slightly faster mechanical response. They also tend to have large power consumption.

- Irreversible-Although significantly limited by the nature of their stroke, these actuators can provide deflection simply upon the addition of water or thermal energy, which is often inherent to the system. They can also provide excellent back pressure control.

\subsubsection{Electro- and magneto-kinetic pump guidelines}

Electro- and magneto-kinetic pumps provide a direct energy transfer to pumping power and generate constant/ steady flows (as compared to oscillatory pumping) due to the continuous addition of energy. However, their performance is often limited by the properties of the selected fluid. Additionally, some pump types require specific surface characteristics for generating flow. This area of micropump research has developed greatly with a number of new possibilities emerging. The following points outline important considerations for selecting or designing dynamic pumps.

4.3.2.1 Flow profiles Since dynamic pumps are generally not pressure-driven, the resulting flow does not always result in parabolic velocity profiles. EO pumps are characterized by a diffuse layer that is largely unaltered by the driving electric field and results in a flow profile that is blunt and plug-like which can reduce sample dispersion 
(Zhang et al. 2007). However, a strong velocity gradient near the wall is beneficial for heat removal. EHD flow profiles are generated by an ionic response to an electric field, which, in turn, drags fluid due to viscous forces. If the ion concentration is strongest near the center of the channel, the flow may be parabolic. However, when the ion concentration is stronger near one of the channel walls, the resulting flow is skew-parabolic. Again, this can be especially attractive in the case where heat is being removed such that a larger velocity gradient occurs near the wall to be cooled.

4.3.2.2 Surface requirement In the case of electroosmotic flow (e.g., using silica-based ceramics), surface silanol groups deprotonate leaving a negative surface charge. Such a configuration requires specific surface material properties to provide a charged surface during operation. However, anionic and cationic polymer coatings have been used to satisfy this requirement (Joo et al. 2007).

4.3.2.3 Fluid properties In electrokinetic devices, the fluid itself plays a critical role in generating flow. Thus flexibility of fluids selection is somewhat limited to those that can provide the necessary properties for actuation. EHD pumps rely critically on electrical properties (specifically permittivity and conductivity). Desired conductivity is usually between $10^{-14}$ and $10^{-9} \mathrm{~S}$ which limits fluid selection. Non-conducting or non-ionic fluids are commonly used (Woias 2005). However, pumping with higher-conductivity fluids with electrokinetic methods has been investigated (Fuhr et al. 1992; Wu et al. 2007). Electroosmotic pumps require a certain $\mathrm{pH}$ when deprotonation of the surface is used to create the electric double layer. The disadvantage of AC electro-osmotic pumping is the inability to pump liquids with ion strengths below $10^{-5} \mathrm{M}$ or above $10^{-2} \mathrm{M}$ (Hansen et al. 2007).

4.3.2.4 No moving parts Many dynamic pump types are comprised of simple structures without moving parts. This is because the motion typically required to generate fluid motion comes from both static and dynamic potentials rather than dynamic mechanical motion.

\subsubsection{Bi-directional capability Flexibility in flow} direction is generally easily provided by electrically actuated pumps simply by changing polarity or traveling wave direction. Further, electrically driven micropumps have very short response times, which can be attractive for timesensitive applications. In cell/particle separation techniques, this rapid response is often exploited in order for particle differentiation (Joo et al. 2007).

4.3.2.6 Pressure Generally speaking, MHD pumps have low pressures whereas EO pumps generate high pressures. High-pressure EO pumps are commonly achieved by filling the channel with a densely packed particle. However, the tortuous flow path through packed particle beds lead to flow mixing in the pump section and may be undesirable depending on the location of the pump in the flow loop or flow path. Further, not all dynamic pump types have wellcharacterized back pressures.

4.3.2.7 Joule heating Joule heating can be significant in the fluid when large currents flow through the liquid as can be the case for EO and MHD pumps. Particle packing can help alleviate this for EO pumps. Joule heating must be considered in MHD pumps has been a topic of research, especially where the application involves a thermally active environment (Patel and Kassegne 2007). Channel width (aspect ratio) has a strong effect on temperature distributions in MHD pumps (Duwairi and Abdullah 2007).

\section{Concluding remarks}

Micropumping techniques that have been reported in very recent years are described and compared in this review. The applicability of these pumping mechanisms for a wide range of applications has been assessed with attention drawn to limitations of the various technologies. Benefits and drawbacks of valve types used in mechanical displacement pumps are also discussed. More than 100 new papers that have appeared in the literature since previous comprehensive reviews were published are included along with references to other important materials, fabrication, and application information. Quantitative comparison of the pumping technologies is provided in tabular form along with extensive qualitative discussion.

Displacement diaphragm pumps as a technology has matured significantly, with most of the research being conducted on various types of actuation as opposed to significant component changes. Diaphragm micropumps are generally characterized by low power requirements, except for those using thermal actuation (bubble and thermopneumatic pumps) or magnetic actuation (electromagnetic or MHD pumps). Diaphragm materials such as PVDF and polymer-metal composites have been developed with an emphasis on reducing the power or voltage requirement. Additionally, irreversible diaphragm pumps used as single-stroke pumping mechanisms may have a 
significant advantage for use in disposable devices as they require little or no power (depending on the device environment and operating conditions).

Electro-kinetic pump types continue to be developed, but their operation and performance are challenging to model and quantify given that the flows are often generated using multi-physical interactions. Regardless, they do offer great miniaturization potential, generally do not require valves, and the understanding of these mechanisms has improved in recent years. Emerging AC electroosmotic pumps have generated high velocities at low voltages, but there is still work to be done for characterizing these pumps.

A comparison of various actuation mechanisms reveals that pumps with high flow rates per unit area include piezoelectric diaphragm pumps, induction- and injection-type EHD, rotary-disk viscous, and EO pumps. Further, vibrating diaphragm and EO pumps also can produce high-back pressures. Depending on the application, systems can be scaled up or down to meet specific requirements of operation. Further, combination of pumping mechanisms in "hybrid" devices could provide the desired flow rates and back pressures to tune them to a wide range of applications.

Acknowledgments The authors acknowledge financial support for this work from members of the Cooling Technologies Research Center (http://www.ecn.purdue.edu/CTRC), a National Science Foundation Industry/University Cooperative Research Center at Purdue University.

\section{References}

Abdelgawad M, Hassan I, Esmail N, Phutthavong P (2005) Numerical investigation of multistage viscous micropump configurations. J Fluids Eng Trans ASME 127(4):734-742

Al-Halhouli AT, Al-Salaymeh A, Kilani MI, Buttgenbach S (2007) Numerical investigation of the effect of spiral curvature on the flow field in a spiral channel viscous micropump. Microfluidics Nanofluidics 3(5):537-546

Andersson H, Van den Berg A (2003) Microfluidic devices for cellomics: a review. Sensors Actuat B Chem 92(3):315

Armani D, Liu C, Aluru N (1999) Re-configurable fluid circuits by PDMS elastomer micromachining. In: Proceedings of the IEEE micro electro mechanical systems (MEMS), Orlando, pp 222 227

Astle AA, Kim HS, Bernal LP, Najafi K, Washabaugh PD (2007) Theoretical and experimental performance of a high frequency gas micropump. Sensors Actuat A Phys 134(1):245-256

Bahadur V, Garimella SV (2006) An energy-based model for electrowetting-induced droplet actuation. J Micromech Microeng 16(8):1494-1503

Bahadur V, Garimella SV (2007) Electrowetting-based control of static droplet states on rough surfaces. Langmuir 23(9):4918 4924

Baroud CN, Delville J-P, Wunenburger R (2005) Laser-actuated microfluidic building blocks. In: Proceedings of SPIE-the international society for optical engineering, optical trapping and optical micromanipulation II, San Diego, vol 5930, pp 1-8
Blanchard D, Ligrani P, Gale B (2005) Single-disk and double-disk viscous micropumps. Sensors Actuat A Phys 122(1 SPEC ISS): $149-158$

Boden R, Lehto M, Simu U, Thornell G, Hjort K, Schweitz J-A (2006) A polymeric paraffin actuated high-pressure micropump. Sensors Actuat A Phys 127(1):88-93

Brask A, Goranovic G, Jensen MJ, Bruus H (2005) A novel electroosmotic pump design for nonconducting liquids: theoretical analysis of flow rate-pressure characteristics and stability. J Micromech Microeng 15(4):883-891

Broderick SL, Webb BW, Maynes D (2005) Thermally developing electro-osmotic convection in microchannels with finite debyelayer thickness. Numer Heat Transfer A Appl 48(10):941-964

Chang H-T, Lee C-Y, Wen C-Y (2007) Design and modeling of electromagnetic actuator in mems-based valveless impedance pump. Microsyst Technol 13(11-12):1615-1622

Chen F, Li B, Sullivan TD, Gonzalez CL, Muzzy CD, Lee HK, Levy MD, Dashiell MW, Kolodzey J (2000) Influence of underlying interlevel dielectric films on extrusion formation in aluminum interconnects. J Vacuum Sci Technol B Microelectr Nanometer Struct 18(6):2826-2834

Chen L, Wang H, Ma J, Wang C, Guan Y (2005a) Fabrication and characterization of a multi-stage electroosmotic pump for liquid delivery. Sensors Actuat B Chem 104(1):117-123

Chen Z, Wang P, Chang H-C (2005b) An electro-osmotic micropump based on monolithic silica for micro-flow analyses and electro-sprays. Anal Bioanalyt Chem 382(3):817-824

Chen SC, Cheng CH, Lin YC (2007) Analysis and experiment of a novel actuating design with a shear mode PZT actuator for microfluidic application. Sensors Actuat APhys 135(1):1-9

Cozma A, Puers B (1995) Characterization of the electrostatic bonding of silicon and pyrex glass. J Micromech Microeng 5(2):98-102

Cui Q, Liu C, Zha XF (2007) Study on a piezoelectric micropump for the controlled drug delivery system. Microfluidics Nanofluidics 3(4):377-390

da Silva AK, Kobayashi MH, Coimbra CFM (2007) Optimal theoretical design of 2-D microscale viscous pumps for maximum mass flow rate and minimum power consumption. Int $\mathbf{J}$ Heat Fluid Flow 28(3):526-536

Darabi J, Wang H (2005) Development of an electrohydrodynamic injection micropump and its potential application in pumping fluids in cryogenic cooling systems. J Microelectromech Syst 14(4):747-755

Darabi J, Rada M, Ohadi M, Lawler J (2002) Design, fabrication, and testing of an electrohydrodynamic ion-drag micropump. J Microelectromech Syst 11(6):684-690

Debesset S, Hayden CJ, Dalton C, Eijkel JCT, Manz A (2004) An AC electroosmotic micropump for circular chromatographic applications. Lab On A Chip 4:396-400

Dissanayake DW, Tikka AC, Al-Sarawi SF, Abbott D (2007) Radio frequency controlled microvalve for biomedical applications. In: Proceedings of SPIE - the international society for optical engineering, Adelaide, Australia, 6413:64130D

Doll A, Heinrichs M, Goldschmidtboeing F, Schrag HJ, Hopt UT, Woias P (2006) A high performance bidirectional micropump for a novel artificial sphincter system. Sensors Actuat A Phys 130131:445-453

Duffy DC, McDonald JC, Schueller OJA, Whitesides GM (1998) Rapid prototyping of microfluidic systems in poly(dimethylsiloxane). Analyt Chem 70(23):4974-4984

Duwairi H, Abdullah M (2007) Thermal and flow analysis of a magnetohydrodynamic micropump. Microsyst Technol 13(1):33-39

Eddings MA, Gale BK (2006) A PDMS-based gas permeation pump for on-chip fluid handling in microfluidic devices. J Micromech Microeng 16(11):2396-2402 
Fan B, Song G, Hussain F (2005) Simulation of a piezoelectrically actuated valveless micropump. Smart Mater Struct 14(2):400405

Fang J, Wang K, Bohringer KF (2006) Self-assembly of PZT actuators for micropumps with high process repeatability. J Microelectromech Syst 15(4):871-878

Faulkner D, Ward C, Gilbuena D, Shekarriz R, Forster FK (2006) Fixed valve piezoelectric micropump for miniature thermal management module. In: Proceedings of ASME fluids engineering division summer meeting 2006, FEDSM2006, Miami, vol 2, pp 843-848

Felten M, Geggier P, Jager M, Duschl C (2006) Controlling electrohydrodynamic pumping in microchannels through defined temperature fields. Phys Fluids 18(5):051707

Feng G-H, Kim ES (2005) Piezoelectrically actuated dome-shaped diaphragm micropump. J Microelectromech Syst 14(2):192-199

Fuhr G, Hagedorn R, Muller T, Benecke W, Wagner B (1992) Microfabricated electrohydrodynamic (EHD) pumps for liquids of higher conductivity. J Microelectromech Syst 1(3):141-146

Gamboa AR, Morris CJ, Forster FK (2005) Improvements in fixedvalve micropump performance through shape optimization of valves. J Fluids Eng Trans ASME 127(2):339-346

Garcia-Sanchez P, Ramos A, Green NG, Morgan H (2006) Experiments on $\mathrm{AC}$ electrokinetic pumping of liquids using arrays of microelectrodes. IEEE Trans Dielectrics Electrical Insulation 13(3):670-677

Garimella SV, Singhal V (2004) Single-phase flow and heat transport and pumping considerations in microchannel heat sinks. Heat Transfer Eng 25(1):15-25

Garimella SV, Singhal V, Liu D (2006) On-chip thermal management with microchannel heat sinks and integrated micropumps. Proc IEEE 94(8): 1534-1548

Geipel A, Doll A, Jantscheff P, Esser N, Massing U, Woias P, Goldschmidtboeing F (2007) A novel two-stage backpressureindependent micropump: modeling and characterization. J Micromech Microeng 17(5):949-959

Go DB, Garimella SV, Fisher TS, Mongia RK (2007) Ionic winds for locally enhanced cooling. J Appl Phys 102(5):053302

Goldschmidtboing F, Doll A, Heinrichs M, Woias P, Schrag HJ, Hopt UT (2005) A generic analytical model for micro-diaphragm pumps with active valves. J Micromech Microeng 15(4):673683

Good BT, Bowman CN, Davis RH (2007) A water-activated pump for portable microfluidic applications. J Colloid Interface Sci 305(2):239-249

Goulpeau J, Trouchet D, Ajdari A, Tabeling P (2005) Experimental study and modeling of polydimethylsiloxane peristaltic micropumps. J Appl Phys 98(4):044914

Grzybowski BA, Brittain ST, Whitesides GM (1999) Thermally actuated interferometric sensors based on the thermal expansion of transparent elastomeric media. Rev Sci Instrum 70(4):20312037

Haeberle S, Schmitt N, Zengerle R, Ducree J (2007) Centrifugomagnetic pump for gas-to-liquid sampling. Sensors Actuat A Phys 135(1):28-33

Haik Y, Kilani M, Hendrix J, Rifai OA, Galambos P (2007) Flow field analysis in a spiral viscous micropump. Microfluidics Nanofluidics 3(5):527-535

Hansen TS, West K, Hassager O, Larsen NB (2007) An all-polymer micropump based on the conductive polymer poly(3,4-ethylenedioxythiophene) and a polyurethane channel system. J Micromech Microeng 17(5):860-866

Homsy A, Linder V, Lucklum F, de Rooij NF (2007) Magnetohydrodynamic pumping in nuclear magnetic resonance environments. Sensors and Actuat B Chem 123(1):636-646
Hu JS, Chao CYH (2007) Numerical study of electroosmotic (EO) flow in microfabricated EO pump with overlapped electrical double layer (EDL). Int J Refrigeration 30(2):290-298

Huang C-W, Lee G-B (2007) A microfluidic system for automatic cell culture. J Micromech Microeng 17(7):1266-1274

Huang C-W, Huang S-B, Lee G-B (2006a) Pneumatic micropumps with serially connected actuation chambers. J Micromech Microeng 16(11):2265-2272

Huang S-C, Lee G-B, Chien F-C, Chen S-J, Chen W-J, Yang M-C (2006b) A microfluidic system with integrated molecular imprinting polymer films for surface plasmon resonance detection. J Micromech Microeng 16(7):1251-1257

Hwang T, Popa D, Sin J, Stephanou HE, Leonard EM (2004) BCB wafer bonding for microfluidics. In: Proceedings of SPIE-the international society for optical engineering, micromachining and microfabrication process technology IX, San Jose, vol 5342, pp 82-191

Ilavsky J, Berndt CC (1998) Thermal expansion properties of metallic and cermet coatings. Surface Coatings Technol 102(1-2):19-24

Inman W, Domansky K, Serdy J, Owens B, Trumper D, Griffith LG (2007) Design, modeling and fabrication of a constant flow pneumatic micropump. J Micromech Microeng 17(5):891-899

Iverson BD, Maynes D, Webb BW (2004) Thermally developing electroosmotic convection in rectangular microchannels with vanishing Debye-layer thickness. J Thermophys Heat Transfer 18(4):486-493

Izzo I, Accoto D, Menciassi A, Schmitt L, Dario P (2007) Modeling and experimental validation of a piezoelectric micropump with novel no-moving-part valves. Sensors Actuat A Phys 133(1):128-140

Jang L-S, Li Y-J, Lin S-J, Hsu Y-C, Yao W-S, Tsai M-C, Hou C-C (2007) A stand-alone peristaltic micropump based on piezoelectric actuation. Biomed Microdevices 9(2):185-194

Jeong OC, Park SW, Yang SS, Pak JJ (2005) Fabrication of a peristaltic PDMS micropump. Sensors Actuat A Phys 123$124: 453$

Joo S, Chung TD, Kim HC (2007) A rapid field-free electroosmotic micropump incorporating charged microchannel surfaces. Sensors Actuat B Chem 123(2):1161-1168

Jung J-Y, Kwak H-Y (2007) Fabrication and testing of bubble powered micropumps using embedded microheater. Microfluidics Nanofluidics 3(2):161-169

Kang Y, Tan SC, Yang C, Huang X (2007) Electrokinetic pumping using packed microcapillary. Sensors Actuat A Phys 133(2):375-382

Kilani MI, Al-Salaymeh A, Al-Halhouli AT (2006) Effect of channel aspect ratio on the flow performance of a spiral-channel viscous micropump. J Fluids Eng 128(3):618-627

Kim J-H, Na K-H, Kang CJ, Kim Y-S (2005a) A disposable thermopneumatic-actuated micropump stacked with PDMS layers and ITO-coated glass. Sensors Actuat A Phys 120(2):365-369

Kim YS, Kim JH, Na KH, Rhee K (2005b) Experimental and numerical studies on the performance of a polydimethylsiloxane valveless micropump. Proc Inst Mech Eng C J Mech Eng Sci 219(10):1139-1145

Kim E-G, Oh J-G, Choi B (2006) A study on the development of a continuous peristaltic micropump using magnetic fluids. Sensors Actuat A Phys 128(1):43-51

Kovacs GTA (1998) Micromachined transducers sourcebook. McGraw-Hill, Boston

Laser DJ, Santiago JG (2004) A review of micropumps. J Micromech Microeng 14(6):35-64

Lee DE, Soper S, Wang W (2007) Fabrication of a microfluidic system with integrated electrochemical pump and valves. In: 
Proceedings of SPIE- the international society for optical engineering, San Jose, vol 6465, pp 64650B

Lee S, Kim KJ (2006) Design of IPMC actuator-driven valve-less micropump and its flow rate estimation at low Reynolds numbers. Smart Mater Struct 15(4):1103-1109

Lee S, Kim KJ, Park HC (2005) Design and performance analysis of a novel IPMC-driven micropump, San Diego, vol 5759, pp 439446

Lei KF, Law WC, Suen Y-K, Li WJ, Yam Y, Ho HP, Kong S-K (2007) A vortex pump-based optically-transparent microfluidic platform for biotech and medical applications. Proc Inst Mech Eng H J Eng Med 221(2):129-141

Lin C-W, Jang J-Y (2005) 3D numerical micro-cooling analysis for an electrohydrodynamic micro-pump. Sensors Actuat A Phys 122(1 SPEC ISS):167-176

Lin Q, Yang B, Xie J, Tai Y-C (2007) Dynamic simulation of a peristaltic micropump considering coupled fluid flow and structural motion. J Micromech Microeng 17(2):220-228

Liu RH, Lodes MJ, Nguyen T, Siuda T, Slota M, Fuji HS, McShea A (2006a) Validation of a fully integrated microfluidic array device for influenza A subtype identification and sequencing. Anal Chem 78(12):4184-4193

Liu RH, Nguyen T, Schwarzkopf K, Fuji HS, Petrova A, Siuda T, Peyvan K, Bizak M, Danley D, McShea A (2006b) Fully integrated miniature device for automated gene expression DNA microarray processing. Anal Chem 78(6):1980-1986

Loverich J, Kanno I, Kotera H (2007) Single-step replicable microfluidic check valve for rectifying and sensing low Reynolds number flow. Microfluidics Nanofluidics 3(4):427-435

Luginbuhl P, Collins SD, Racine GA, Gretillat MA, de Rooij NF, Brooks KG, Setter N (1998) Ultrasonic flexural Lamb-wave actuators based on PZT thin film. Sensors Actuat A Phys 64(1):41-49

Machauf A, Nemirovsky Y, Dinnar U (2005) A membrane micropump electrostatically actuated across the working fluid. J Micromech Microeng 15(12):2309-2316

Mahajan R, Chiu C-P, Chrysler G (2006) Cooling a microprocessor chip. Proc IEEE 94(8):1476-1486

Maruo S, Inoue H (2006) Optically driven micropump produced by three-dimensional two-photon microfabrication. Appl Phys Lett 89(14): 144101

Matsumoto S, Maeda R, Klein A (1999) Characterization of a valveless micropump based on liquid viscosity. Microscale Thermophys Eng 3(1):31-42

Matteucci M, Perennes F, Marmiroli B, Miotti P, Vaccari L, Gosparini A, Turchet A, Di Fabrizio E (2006) Compact micropumping system based on LIGA fabricated microparts. Microelectr Eng 83(4-9):1288-1290

MatWeb (2007) MatWeb material property data in http://www. matweb.com/, accessed November 28, 2007

Melcher JR (1981) Continuum electromechanics. MIT Press, Cambridge

MEMSnet (2007) MEMS and nanotechnology clearinghouse material index in http://www.memsnet.org/material/, accessed November 28,2007

Moghaddam S, Ohadi MM (2005) Effect of electrode geometry on performance of an EHD thin-film evaporator. J Microelectromech Syst 14(5):978-986

Morganti E, Fuduli I, Montefusco A, Petasecca M, Pignatel GU (2005) SPICE modelling and design optimization of micropumps. Int J Environ Anal Chem 85(9-11):687-698

Mpholo M, Smith CG, Brown ABD (2003) Low voltage plug flow pumping using anisotropic electrode arrays. Sensors Actuat B Chem 92(3):262-268

Mugele F, Baret J-C (2005) Electrowetting: from basics to applications. J Phys Condensed Matter 17(28):705-774
Nagel JJ, Mikhail G, Noh H, Koo J (2006) Magnetically actuated micropumps using an Fe-PDMS composite membrane. In: Proceedings of SPIE - the international society for optical engineering, smart structures and materials 2006, San Diego, vol 6172, pp 617213

Nakano M, Katsura S, Touchard GG, Takashima K, Mizuno A (2007) Development of an optoelectrostatic micropump using a focused laser beam in a high-frequency electric field. IEEE Trans Ind Appl 43(1):232-237

Nguyen N-T, Huang X (2005) Development of a peristaltic pump in printed circuit boards. J Micromechatronics 3(1):1-13

Nguyen N-T, Meng AH, Black J, White RM (2000) Integrated flow sensor for in situ measurement and control of acoustic streaming in flexural plate wave micropumps. Sensors Actuat A Phys 79(2):115-121

Nguyen N-T, Huang X, Chuan TK (2002) MEMS-micropumps: a review. J Fluids Eng Trans ASME 124(2):384-392

Nguyen T-T, Goo NS, Yoon YS, Yoon KJ (2006) A novel lightweight piezo-composite actuator micropump. In: Proceedings of SPIEthe international society for optical engineering, smart structures and materials 2006, San Diego, vol 6172, pp 617212

Oberhammer J, Niklaus F, Stemme G (2003) Selective wafer-level adhesive bonding with benzocyclobutene for fabrication of cavities. Sensors Actuat A Phys 105(3):297-304

Olesen LH, Bruus H, Ajdari A (2006) Ac electrokinetic micropumps: the effect of geometrical confinement, Faradaic current injection, and nonlinear surface capacitance. Phys Rev E 73(5):056313

Pan T, McDonald SJ, Kai EM, Ziaie B (2005) A magnetically driven PDMS micropump with ball check-valves. J Micromech Microeng 15(5):1021-1026

Patel V, Kassegne SK (2007) Electroosmosis and thermal effects in magnetohydrodynamic (MHD) micropumps using 3D MHD equations. Sensors Actuat B Chem 122(1):42-52

Piyasena ME, Lopez GP, Petsev DN (2006) An electrokinetic cell model for analysis and optimization of electroosmotic microfluidic pumps. Sensors Actuat B Chem 113(1):461-467

Samel B, Chretien J, Yue R, Griss P, Stemme G (2007a) Wafer-level process for single-use buckling-film microliter-range pumps. J Microelectromech Syst 16(4):795-801

Samel B, Griss P, Stemme G (2007b) A thermally responsive PDMS composite and its microfluidic applications. J Microelectromech Syst 16(1):50-57

SCS Coatings (2007) Parylene specifications and properties in http://www.scscoatings.com/parylene_knowledge/ specifications.aspx, accessed November 28, 2007

Seyed-Yagoobi J (2005) Electrohydrodynamic pumping of dielectric liquids. J Electrostat 63(6-10):861-869

Shin DD, Mohanchandra KP, Carman GP (2005) Development of hydraulic linear actuator using thin film SMA. Sensors Actuat A Phys 119(1):151-156

Sim W, Oh J, Choi B (2006) Fabrication, experiment of a microactuator using magnetic fluid for micropump application. Microsyst Technol 12(12):1085-1091

Singhal V, Garimella SV (2005a) Influence of bulk fluid velocity on the efficiency of electrohydrodynamic pumping. J Fluids Eng 127(3):484-494

Singhal V, Garimella SV (2005b) A novel valveless micropump with electrohydrodynamic enhancement for high heat flux cooling. IEEE Trans Adv Packaging 28(2):216-230

Singhal V, Garimella SV (2007) Induction electrohydrodynamics micropump for high heat flux cooling. Sensors Actuat A Phys 134(2):650-659

Singhal V, Garimella SV, Murthy JY (2004a) Low Reynolds number flow through nozzle-diffuser elements in valveless micropumps. Sensors Actuat A Phys 113(2):226-235 
Singhal V, Garimella SV, Raman A (2004b) Microscale pumping technologies for microchannel cooling systems. Appl Mech Rev 57(1-6):191-221

Song WH, Lichtenberg J (2005) Thermo-pneumatic, single-stroke micropump. J Micromech Microeng 15(8):1425-1432

Stemme E, Stemme G (1993) Valveless diffuser/nozzle-based fluid pump. Sensors Actuat A Phys 39(2):159-167

Su Y, Chen W, Cui F, Zhang W (2005) Analysis and fabrication process of an electromagnetically actuated valveless micropump with two parallel flexible diaphragms. Proc Inst Mech Eng C J Mech Eng Sci 219(9):1007-1014

Suzuki H (2006) Stimulus-responsive gels: Promising materials for the construction of micro actuators and sensors. J Intell Mater Syst Struct 17(12):1091-1097

Tang KC, Liao E, Ong WL, Wong JDS, Agarwal A, Nagarajan R, Yobas L (2006) Evaluation of bonding between oxygen plasma treated polydimethyl siloxane and passivated silicon. In: Journal of Physics: Conference Series, International MEMS Conference 2006, Singapore, vol 34, pp 155-161

Tesla N (1920) Valvular conduit. U.S. Patent No. 1,329,559

Tracey MC, Johnston ID, Davis JB, Tan CKL (2006) Dual independent displacement-amplified micropumps with a single actuator. J Micromech Microeng 16(8):1444-1452

Truckenmuller R, Cheng Y, Ahrens R, Bahrs H, Fischer G, Lehmann J (2006) Micro ultrasonic welding: joining of chemically inert polymer microparts for single material fluidic components and systems. Microsyst Technol 12(10-11):1027-1029

Urbanski JP, Thorsen T, Levitan JA, Bazant MZ (2006) Fast ac electro-osmotic micropumps with nonplanar electrodes. Appl Phys Lett 89(14):143508

Vajandar SK, Xu D, Markov DA, Wikswo JP, Hofmeister W, Li D (2007) $\mathrm{SiO}_{2}$-coated porous anodic alumina membranes for high flow rate electroosmotic pumping. Nanotechnology 18(27): 275705

Vijendran S, Smith CG, Mpholo MI (2006) Multi-directional electrokinetic pumping for efficient transport and mixing in biochip applications. In: Proceedings of SPIE-the international society for optical engineering, microfluidics, biomems, and medical microsystems IV, San Jose, vol 6112, pp 61120

Wallis G, Pomerantz DI (1969) Field assisted glass-metal sealing. J Appl Phys 40(10):3946-3949

Woias P (2005) Micropumps-past, progress and future prospects. Sensors Actuat B Chem 105(1):28-38

Wolff A, Perch-Nielsen IR, Larsen UD, Friis P, Goranovic G, Poulsen CR, Kuttera JP, Telleman P (2003) Integrating advanced functionality in a microfabricated high-throughput fluorescentactivated cell sorter. Lab On A Chip 3(1):22

Wu J (2006) Biased AC electro-osmosis for on-chip bioparticle processing. IEEE Trans Nanotechnol 5(2):84-88

Wu J, Lian M, Yang K (2007) Micropumping of biofluids by alternating current electrothermal effects. Appl Phys Lett 90(23):234103
Xu T-B, Su J (2005) Development, characterization, and theoretical evaluation of electroactive polymer-based micropump diaphragm. Sensors Actuat A (Physical) 121(1):267-274

Yamahata C, Chastellain M, Parashar VK, Petri A, Hofmann H, Gijs MAM (2005a) Plastic micropump with ferrofluidic actuation. J Microelectromech Syst 14(1):96-102

Yamahata C, Lacharme F, Burri Y, Gijs MAM (2005b) A ball valve micropump in glass fabricated by powder blasting. Sensors Actuat B Chem 110(1):1-7

Yamahata C, Lotto C, Al-Assaf E, Gijs MAM (2005c) A PMMA valveless micropump using electromagnetic actuation. Microfluidics Nanofluidics 1(3):197-207

Yang S-Y, Hsiung S-K, Hung Y-C, Chang C-M, Liao T-L, Lee G-B (2006) A cell counting/sorting system incorporated with a microfabricated flow cytometer chip. Meas Sci Technol 17(7):2001-2009

Yin Z, Prosperetti A (2005a) 'Blinking bubble' micropump with microfabricated heaters. J Micromech Microeng 15(9):16831691

Yin Z, Prosperetti A (2005b) A microfluidic 'blinking bubble' pump. J Micromech Microeng 15(3):643-651

Yokota K, Sato K, Itoh M (2006) Model experiment, numerical simulation and theoretical analysis on the characteristics of a viscous micropump using a cylindrical rotor in a rectangular duct. JSME Int J Ser B Fluids Thermal Eng 49(2):393-400

Yoon JS, Choi JW, Lee IH, Kim MS (2007) A valveless micropump for bidirectional applications. Sensors Actuat A Phys 135(2):833-838

Yufeng S, Wenyuan C, Feng C, Weiping Z (2006) Electro-magnetically actuated valveless micropump with two flexible diaphragms. Int J Adv Manufact Technol 30(3-4):215-220

Yun K-S, Cho I-J, Bu J-U, Kim C-J, Yoon E (2002) A surface-tension driven micropump for low-voltage and low-power operations. J Microelectromech Syst 11(5):454-461

Zeng S, Chen CH, Mikkelsen JC Jr, Santiago JG (2001) Fabrication and characterization of electroosmotic micropumps. Sensors Actuat B Chem 79(2-3): 107

Zengerle R, Richter A, Sandmaier H (1992) A micro membrane pump with electrostatic actuation. In: Proceedings of the IEEE Micro Electro Mechanical Systems Workshop, Travemuende, Germany, pp 19-24

Zhang HJ, Qiu CJ (2006) Characterization and MEMS application of low temperature $\mathrm{TiNi}(\mathrm{Cu})$ shape memory thin films. Mater Sci Eng A 438-440:1106-1109

Zhang T, Wang Q-M (2005) Valveless piezoelectric micropump for fuel delivery in direct methanol fuel cell (DMFC) devices. J Power Sources 140(1):72-80

Zhang C, Xing D, Li Y (2007) Micropumps, microvalves, and micromixers within PCR microfluidic chips: advances and trends. Biotechnol Adv 25(5):483-514 